\title{
Oxytocin Receptors Are Expressed by Glutamatergic Prefrontal Cortical Neurons That Selectively Modulate Social Recognition
}

\author{
Yalun Tan, ${ }^{1 \star}$ Sarthak M. Singhal, ${ }^{1 \star}$ CScott W. Harden, ${ }^{1}$ Karlena M. Cahill, ${ }^{1}$ Dan-Tam M. Nguyen, ${ }^{1}$ \\ (ㄴ) Luis M. Colon-Perez, ${ }^{2}$ Todd J. Sahagian, ${ }^{1}$ Jeffrey S. Thinschmidt, ${ }^{1}{ }^{\oplus}$ Annette D. de Kloet, ${ }^{3,4}{ }^{-}$Marcelo Febo, ${ }^{2,4}$ \\ CCharles J. Frazier, ${ }^{1}$ and Eric G. Krause ${ }^{1,4}$ \\ ${ }^{1}$ Department of Pharmacodynamics, College of Pharmacy, ${ }^{2}$ Department of Psychiatry, College of Medicine, ${ }^{3}$ Department of Physiology and Functional \\ Genomics, College of Medicine, and ${ }^{4} \mathrm{McKnight}$ Brain Institute, University of Florida, Gainesville, Florida 32611
}

Social recognition, the ability to recognize individuals that were previously encountered, requires complex integration of sensory inputs with previous experience. Here, we use a variety of approaches to discern how oxytocin-sensitive neurons in the PFC exert descending control over a circuit mediating social recognition in mice. Using male mice with Cre-recombinase directed to the oxytocin receptor gene (Oxtr), we revealed that oxytocin receptors (OXTRs) are expressed on glutamatergic neurons in the PFC, optogenetic stimulation of which elicited activation of neurons residing in several mesolimbic brain structures. Optogenetic stimulation of axons in the BLA arising from OXTR-expressing neurons in the PFC eliminated the ability to distinguish novel from familiar conspecifics, but remarkably, distinguishing between novel and familiar objects was unaffected. These results suggest that an oxytocin-sensitive PFC to BLA circuit is required for social recognition. The implication is that impaired social memory may manifest from dysregulation of this circuit.

Key words: anxiety; autism; basolateral amygdala; bed nucleus of the stria terminalis; nucleus accumbens; social novelty

Significance Statement

Using mice, we demonstrate that optogenetic activation of the neurons in the PFC that express the oxytocin receptor gene (Oxtr) impairs the ability to distinguish between novel and familiar conspecifics, but the ability to distinguish between novel and familiar objects remains intact. Subjects with autism spectrum disorders (ASDs) have difficulty identifying a person based on remembering facial features; however, ASDs and typical subjects perform similarly when remembering objects. In subjects with ASD, viewing the same face increases neural activity in the PFC, which may be analogous to the optogenetic excitation of oxytocin receptor (OXTR) expressing neurons in the PFC that impairs social recognition in mice. The implication is that overactivation of OXTR-expressing neurons in the PFC may contribute to ASD symptomology.

\section{Introduction}

Social recognition or the ability to distinguish between familiar and novel conspecifics is critical for establishing preferential social interactions that enhance survival (Brennan and Kendrick, 2006). Individuals with autism spectrum disorders (ASDs) have difficulties identifying faces of novel conspecifics from those pre-

\footnotetext{
Received Nov. 15, 2018; revised Feb. 6, 2019; accepted Feb. 11, 2019.

Author contributions: Y.T., S.M.S., S.W.H., L.M.C.-P., A.D.d.K., M.F., C.J.F., and E.G.K. designed research; Y.T., S.M.S., S.W.H., K.M.C., D.-T.M.N., L.M.C.-P., T.J.S., J.S.T., A.D.d.K., M.F., C.J.F., and E.G.K. performed research; Y.T., S.M.S., S.W.H., A.D.d.K., M.F., C.J.F., and E.G.K. analyzed data; Y.T., M.F., C.J.F., and E.G.K. wrote the first draft of the paper; S.W.H., L.M.C.-P., A.D.d.K., M.F., C.J.F., and E.G.K. edited the paper; C.J.F. and E.G.K. wrote the paper.

This work was supported by R01HL122494 and R01MH104641.

The authors declare no competing financial interests.

*Y.T. and S.M.S. contributed equally to this work.

Correspondence should be addressed to Charles J. Frazier at cjfraz@ufl.edu or Eric G. Krause at ekrause@cop.ufl.edu.

https://doi.org/10.1523/JNEUROSCI.2944-18.2019

Copyright $\odot 2019$ the authors
}

viously encountered, indicating that deficits in social recognition may contribute to the symptomology of ASD (Weigelt et al., 2012; Ewbank et al., 2017). In rodents, paradigms that assess social recognition rely on their inherent propensity to shift interest toward novel conspecifics and away from familiar conspecifics when given the opportunity to interact with both (Bluthé et al., 1993; Dantzer, 1998). Analogous to the atypical facial memory that occurs with ASD, this preference for social novelty is absent in mice harboring genetic mutations linked to ASD (Silverman et al., 2010; Zhou et al., 2016). While these deficits in social recognition are well established, the underlying neurobiology is poorly understood.

Oxytocin, a neuropeptide synthesized primarily by the neurons within the paraventricular and supraoptic nuclei of the hypothalamus (Sofroniew, 1983; Swanson and Sawchenko, 1983; Landgraf and Neumann, 2004), has been implicated in both the etiology and alleviation of ASD (Lefevre and Sirigu, 2016; Amadei et al., 2017). Polymorphisms in the oxytocin receptor gene (Oxtr) 
are predictive of ASD and intranasal delivery of oxytocin alleviates symptoms of the disorder (LoParo and Waldman, 2015; Lefevre and Sirigu, 2016). In mammals, oxytocinergic axons and oxytocin receptors (OXTRs) permeate the CNS (Elands et al., 1988; Knobloch et al., 2012; Chini et al., 2017), and recent studies have found that oxytocinergic projections and/or OXTRs serve as phenotypic markers for distinct neuronal circuits that control specific facets of social behavior (Stoop, 2012; Oettl et al., 2016; Amadei et al., 2017; Raam et al., 2017). Clinical and preclinical research indicates that impaired social recognition is associated with structural, neurochemical, and excitatory alterations within the PFC (Zhou et al., 2016; Ewbank et al., 2017). The PFC is innervated by oxytocin-synthesizing neurons (Knobloch et al., 2012), densely expresses OXTRs (Smeltzer et al., 2006; Nakajima et al., 2014; Li et al., 2016), and contributes to a neuronal network mediating social behavior (Tanimizu et al., 2017). Whether OXTRs in the PFC serve as phenotypic markers of specific neurons that orchestrate social recognition and the preference for social novelty remains to be discerned.

In this series of experiments, we test the hypothesis that OXTR-expressing neurons residing in the PFC exert descending control over subcortical nuclei that is necessary for social recognition. Using mice genetically engineered to direct Cre recombinase expression to cells synthesizing OXTR, we conducted neuroanatomical and electrophysiological experiments revealing that neurons in the PFC that synthesize functional OXTRs are GABAergic or glutamatergic, with the latter sending long-range projections to subcortical structures. Subsequent experiments combined use of in vivo optogenetics with fMRI to discover that light-induced excitation of OXTR-expressing neurons in the PFC increased neuronal activity within mesolimbic nuclei implicated in rewarding (Berridge and Kringelbach, 2015), social (Chen and Hong, 2018), and anxiety-like behaviors (Davis et al., 2010). The neuronal activation and axonal innervation observed in the amygdala were particularly robust. In vivo optogenetic activation of OXTR-expressing neurons in the PFC impaired social recognition; however, selective activation of these neurons had no effect on novel object recognition, sociability, anxiety-like behavior, or locomotor activity. In vitro electrophysiological experiments used retrograde neuronal tract tracing and channelrhodopsin- 2 assisted circuit mapping (CRACM) to characterize the connectivity between OXTR-expressing neurons in the PFC and their postsynaptic targets in the BLA. These experiments found that oxytocin depolarizes pyramidal neurons in the PFC that make glutamatergic synapses onto pyramidal neurons and GABAergic interneurons in the BLA. Interestingly, in vivo optogenetic excitation of axons in the BLA that originated from OXTR-expressing neurons residing in the PFC specifically impaired social recognition. Collectively, the results suggest that overactivation of an oxytocin-sensitive PFC to BLA connection impairs social recognition.

\section{Materials and Methods}

Animals. Male heterozygous Oxtr-Cre mice (Mutant Mouse Resource Research Centers, stock \#036545-UCD) on a C57BL/6 background were used in the current study. This mouse line has Cre recombinase in OXTR-expressing cells (i.e., Cre cassettes under control of an specific promoter were inserted into bacterial artificial chromosome). Details of this mouse line can be found at vendor's website (https://www.mmrrc. org/catalog/sds.php?mmrrc_id=36545). Mice were housed individually with standard rodent chow (Teklad 7912, Envigo) and water ad libitum. Housing conditions were temperature $\left(20^{\circ} \mathrm{C}-26^{\circ} \mathrm{C}\right)$ and humidity $(30 \%-70 \%)$ controlled and kept on a 12:12 h light/dark cycle. All procedures were approved by the Institutional Animal Care and Use Committee at the University of Florida.
PFC opsin placement. Cre-inducible adeno-associated virus (AAV)containing viral constructs, AAV2/EF1a-DIO-hChR2(H134r)-eYFP, AAV2/EF1a-DIO-eYFP, or AAV1-CAG-Flex-GCaMP6f-WPRE-SV40 (Dr. Karl Deisseroth at Stanford University; UNC Gene Therapy Center Vector Core; Penn Vector Core) were delivered bilaterally into the prelimbic cortices of Oxtr-Cre mice to study the phenotypes and characteristics of OXTR-expressing neurons. Mice were kept under isoflurane anesthesia and on a heating pad throughout the surgery. Buprenorphine $(0.12 \mathrm{mg} / \mathrm{kg})$ was subcutaneously injected for analgesia. Ophthalmic ointment was applied for corneal protection. After fur removal and skin disinfection, mice were fixed on the stereotaxic frame, and a longitudinal incision was made to expose the skull. Two holes were drilled in the skull using the coordinates from bregma: anterior $2.25 \mathrm{~mm}$, lateral $0.45 \mathrm{~mm}$. Cre-dependent AAV(s) were loaded in pulled glass pipettes (Sutter Instrument, outside diameter: $1.5 \mathrm{~mm}$; inside diameter: $0.86 \mathrm{~mm}$ ) and injected (250-350 $\mu \mathrm{l} /$ per side) into the mPFC (from bregma: anterior 2.25 $\mathrm{mm}$; lateral $0.45 \mathrm{~mm}$; ventral: $-1.4 \mathrm{~mm}$, measured from brain surface) via pressure injection system (PV830 Pneumatic PicoPump, WPI). For mice used in anatomical or electrophysiological studies, the incisions were sutured, and mice were returned to home cages for recovery.

Retrograde tracer injection. Fluoro-Gold (FG), a retrograde tracer that travels back to the soma after being taken up by axonal terminals, was injected in several brain regions to study the projections of OXTRexpressing neurons in the PFC. Dissolved FG was loaded in pulled glass pipettes (Sutter Instrument, outside diameter: $1.0 \mathrm{~mm}$; inside diameter: $0.58 \mathrm{~mm}$ ) and injected into the NAc (anterior $1.6 \mathrm{~mm}$; lateral $1.5 \mathrm{~mm}$; ventral $-4.32 \mathrm{~mm}$ ), bed nucleus of the stria terminalis (BNST, anterior $0.66 \mathrm{~mm}$; lateral $0.75 \mathrm{~mm}$; ventral $-3.85 \mathrm{~mm}$ ), or BLA (anterior -0.94 $\mathrm{mm}$; lateral $3.25 \mathrm{~mm}$; ventral $-4.5 \mathrm{~mm}$ ) via iontophoresis ( $7 \mathrm{~s}$ on/off, 5.0 $\mu \mathrm{A}$, Stoelting).

Red fluorescent microspheres (580/605, $0.04 \mu \mathrm{m}$, Invitrogen F8793, $550-1500 \mathrm{nl}$ ) were injected into the BLA (anterior $-0.5 \mathrm{~mm}$; lateral 2.5-3 $\mathrm{mm}$; ventral $-5 \mathrm{~mm}$ ) to study oxytocin-responsive neurons that project from the PFC to the BLA. Mice were permitted to recover for $48-72 \mathrm{~h}$ before being killed for electrophysiological experiments.

Fiber optics implantation. Separate cohorts of mice were used for in vivo optogenetics to study the behavioral effects of stimulating OXTRexpressing somas in the PFC or their axon terminals in the BLA. After the skull was exposed and the holes were drilled, mice were injected with Cre-dependent AAV2/EF1a-DIO-hChR2(H134r)-eYFP (AAV-ChR2eYFP) while their littermates received control virus AAV2/EF1a-DIOeYFP (AAV-eYFP). Injections bilaterally targeted the PFC using the stereotaxic coordinates described above. After virus injection, two fiber optics (CFLC230-10 ceramic ferrules with FT200UMT fiber, Thorlabs) were implanted bilaterally into either PFC or BLA (for PFC: from bregma, anterior $2.25 \mathrm{~mm}$; lateral $0.45 \mathrm{~mm}$; ventral $-1.2 \mathrm{~mm}$; for BLA: from bregma, anterior $-0.94 \mathrm{~mm}$; lateral $3.25 \mathrm{~mm}$; ventral $-4.25 \mathrm{~mm}$ ). One nylon screw (8L080X093N01, PlasticsOne) was anchored into the skull to provide stability. Super glue and dental cement were used to secure the fiber optics and screw to the skull. After the cement dried, mice were returned to home cages for recovery. Experiments using in vivo optogenetics used the following groups: Oxtr-Cre mice delivered control AAV-eYFP without blue light stimulation (control-NL), Oxtr-Cre mice delivered AAV-ChR2-eYFP without blue light stimulation (ChR2-NL), Oxtr-Cre mice delivered control AAV-eYFP with blue light stimulation (control-BLS), and Oxtr-Cre mice delivered AAV-ChR2-eYFP with blue light stimulation (ChR2-BLS). The number of subjects used for each experiment is indicated in Results and figure legends.

Preexperiment habituation. For cohorts used for behavioral tests, habituation started $3 \mathrm{~d}$ after fiber optic implantation. Mice underwent daily handling for 1 week after surgery, during which they were stroked and touched around the fiber optic implants for $\sim 3$ min per session. A quick assessment was made during the last handling session; if a mouse still exhibited high levels of anxiety-like behavior (incontinence, hyperactivity, etc.), an additional one or two handling sessions were conducted before moving on to the next step. Mice next underwent daily tethering habituation for another week, during which they stayed in their home cages for $30 \mathrm{~min}$ with their implants connected to a fiber optic cable. 
Before each session, home cages were transported to the procedure room (where behavioral tests would take place); and after the end of each session, they were returned to the housing room.

Social interaction and social novelty tests. The social interaction test was conducted $2 \mathrm{~d}$ after the last session of tethering habituation. The social interaction chamber was made from clear Plexiglas with white flooring and was separated into three sections by removable Plexiglas panels (see Fig. 7). The dimensions of the chamber were $60 \times 45 \times 20 \mathrm{~cm}$ (length $\times$ width $\times$ height). Each of the 2 side compartments of the chamber had a wire mesh cup (20 cm tall) placed in the middle. Before testing, each mouse was brought into the procedure room singly and underwent $5 \mathrm{~min}$ of habituation in their home cage with the cable connected. Subsequently, the mouse was brought into in the center (neutral) compartment of the interaction chamber for habituation for another $5 \mathrm{~min}$. During the first phase of the test, an unfamiliar conspecific (same genetic background, same sex and age) was placed under one of the wire mesh cups. The Plexiglas doors were removed, allowing for free access to the entire interaction chamber, and a video camera recorded the movement and behaviors of the test mouse. Ten minutes later, a new, unfamiliar conspecific, the "novel" mouse, was placed under the previously empty cup while the original conspecific, now the "familiar" mouse, remained under the other cup in the other chamber. This phase of behavioral testing was referred to as the social recognition test. The duration of the combined social interaction and social recognition tests was $20 \mathrm{~min}$. During the entire test, laser-generated $473 \mathrm{~nm}$ blue light was delivered to mice in the blue light subgroup but not to mice in the no light subgroup. The blue light stimulation (BLS) pattern for PFC activation was $20 \mathrm{~ms}$ pulses, $15 \mathrm{~Hz}, 75$ pulses/sequence, 12 repeated sequences with $5 \mathrm{~s}$ delay in a $2 \mathrm{~min}$ on/ $2 \mathrm{~min}$ off cycle; for BLA activation was $20 \mathrm{~ms}$ pulses, $15 \mathrm{~Hz}, 75$ pulses/sequence, 24 repeated sequences without delay in a $2 \mathrm{~min}$ on/2 min off cycle. When the test was over, the test mouse was returned to its home cage, and the chamber was thoroughly cleaned with $70 \%$ ethanol to minimize the influence of olfactory clues. The location of the mouse/ empty cup and familiar/novel mice was randomized between test mice, to counteract side preferences.

The social interaction and social novelty tests were recorded by a ceiling-mounted camera and a horizontally placed HD camera. Data, including exploratory and repetitive behaviors, were recorded automatically by TopScan software (CleverSystem) and analyzed manually by Behavioral Observation Research Interactive Software (BORIS, Universita Degli Studi Di Torino).

Novel Object Recognition Test. The novel object recognition test was conducted in the same apparatus used for the social interaction and social novelty tests. Before the testing, each mouse was brought into the procedure room singly. For the first $5 \mathrm{~min}$, the test mouse, with fiber optic cable connected, was given free access to the entire chamber for habituation. When the test started, two identical small red cups were placed in the center of the side chambers, and the test mouse was allowed to freely explore for $10 \mathrm{~min}$. After $10 \mathrm{~min}$, one of the red cups was replaced with a small green or purple bottle. The test mouse was then given another $10 \mathrm{~min}$ to explore the chambers while exploratory behaviors (sniffing, rearing against the objects, and head within $2 \mathrm{~cm}$ toward the object) were recorded. BLS parameters were identical to those described for the social interaction and social novelty tests. Videos were processed and analyzed for exploration time with BORIS software.

Open Field Test. The Open Field Test was conducted in a $45 \times 45 \times 30$ $\mathrm{cm}$ (length $\times$ width $\times$ height) square arena constructed of white plastic boards. Before testing, each mouse was brought into the procedure room singly and underwent $5 \mathrm{~min}$ of habituation in their home cage with cable connected. Upon completion of the habituation period, the mouse was placed in the center of the open field arena. Results, including total distance traveled and duration in center (located in the center of arena with $25 \%$ of total area) or edge, were processed and analyzed by TopScan software (CleverSystem). The total duration of Open Field Test, excluding habituation, was $15 \mathrm{~min}$. BLS parameters for PFC activation was a 3 $\mathrm{min}$ on/3 $\mathrm{min}$ off cycle, and BLA activation was a $2 \mathrm{~min}$ on $/ 3 \mathrm{~min}$ off cycle.

Elevated Plus Maze (EPM) Test. The EPM arena has two opposing open arms and two opposing closed arms, raised $40 \mathrm{~cm}$ above the floor. All arms were $30 \times 5 \mathrm{~cm}$ (length $\times$ width) with white walls $(15 \mathrm{~cm}$ height $)$ and floors. The test started $2 \mathrm{~h}$ after the end of the dark cycle. After $5 \mathrm{~min}$ of habituation within the home cage with the cable connected, the test mouse was placed in the center of the maze and allowed to freely explore the arena. The total duration of the test was $6 \mathrm{~min}$ and $5 \mathrm{~min}$ for activation of the PFC or BLA, respectively. BLS parameters for PFC activation were a $3 \mathrm{~min}$ on $/ 3 \mathrm{~min}$ off cycle for $6 \mathrm{~min}$, but for BLA parameters were a $1 \mathrm{~min}$ on/1 min off cycle for $5 \mathrm{~min}$. Results, including the duration of time spent in exploratory behaviors, number of entries, and distance traveled in open and closed arms (excluding center), were processed and analyzed by TopScan software (CleverSystem).

Tissue collection and sectioning. Two weeks after viral delivery for anatomical or behavioral experiments (in vivo optogenetic stimulation), mice were deeply anesthetized with sodium pentobarbital $(1.56 \mathrm{mg} / \mathrm{g}$, i.p.). Transcardial perfusions were performed by clearing with $0.15 \mathrm{M}$ $\mathrm{NaCl}$ followed by fixation with 4\% PFA (Sigma-Aldrich). Brains were extracted and postfixed in $4 \%$ PFA for $4 \mathrm{~h}$. For mice implanted with fiber optics, dental cement, and the fibers were removed with rongeurs before brain extraction. After postfixation, brains were transferred into $30 \%$ sucrose and stored at $4^{\circ} \mathrm{C}$, until sectioned for either ISH or immunohistochemistry.

Brains for ISH were sectioned at a thickness of $20 \mu \mathrm{m}$ using a cryostat (CM3050S, Leica Microsystems). The cryostat and other tools were cleaned with RNase remover (Thermo Fisher Scientific) and ethanol before use. Tissues were first collected in RNase-free PBS and then mounted on slides (Tissue Path Superfrost Plus Gold Microscope Slides, Thermo Fisher Scientific). Slides were air dried and stored at $-80^{\circ} \mathrm{C}$. Brains for immunohistochemistry experiments were sectioned at $30 \mu \mathrm{m}$ and stored in cryoprotective solution (1 L 0.1 M PBS, $20 \mathrm{~g}$ PVP-40, $600 \mathrm{ml}$ ethylene glycol, $600 \mathrm{~g}$ sucrose) at $-20^{\circ} \mathrm{C}$.

$I S H$. ISH with various probes was performed to examine Cre recombinase expression in Oxtr-Cre mice and the phenotype of OXTRexpressing neurons. Detailed procedures and the reagents are provided as per the manufacturer's instructions (Advanced Cell Diagnostics) and as previously described (de Kloet et al., 2016). Brain sections were first treated with pretreatment IV (Advanced Cell Diagnostics, lot \#2002337) for $20 \mathrm{~min}$ in the dark. After 3 consecutive washes with DEPC-treated water, sections were incubated with probes for Oxtr, Gad 1, or VGlut1 mRNA (working dilution 1:50, Advanced Cell Diagnostics) in probe diluent for $2 \mathrm{~h}$ at $40^{\circ} \mathrm{C}$. The probes contained double $\mathrm{Z}$-shaped probe pairs, which are complementary to target RNA, and thus decreasing nonselective binding. After incubation, sections were rinsed 3 times with wash buffer reagents followed by Amplification steps I, II, III, and IV. After Amplification IV, sections were rinsed 3 times with wash buffer reagent.

Immunohistochemistry. Immunohistochemistry was conducted on its own or immediately after ISH. Brain sections were rinsed 5 times in 50 mM potassium PBS (KPBS) for $5 \mathrm{~min}$, and then blocked in $2 \%$ normal donkey serum and $0.2 \%$ Triton X-100 in KPBS to minimize nonspecific binding. Sections were then incubated with primary antibody in blocking solution overnight at $4^{\circ} \mathrm{C}$. For enhanced yellow fluorescent protein (eYFP) immunohistochemistry, chicken anti-GFP antibody (working dilution 1:1000, Invitrogen) was used; for FG staining, rabbit anti-FG antibody (1:3000, Millipore) was applied; and for HuC/D (neuronal specific marker) staining, mouse anti-HuC/D antibody (1:500, Invitrogen) was used. Sections were rinsed in KPBS and incubated in relevant secondary antibody for $2 \mathrm{~h}$ the next day. After the last set of KPBS rinses, sections were mounted, air dried overnight, coverslipped with polyvinyl alcohol mounting medium, and stored at $4^{\circ} \mathrm{C}$.

Image capture. Images were captured and analyzed using an AxioImager fluorescent Apotome microscope (Carl Zeiss) and Axiovision version 4.8.2 software. Each channel was measured and exposure time adjusted for optimal settings. For ISH, negative control slides were used for background subtraction: the detection threshold for the negative control slide was chosen as the exposure and adjustment settings for all samples, including negative/positive controls and experimental slides. ImageJ (Schneider et al., 2012) was used for making mosaics.

Optogenetic fMRI. Functional images were collected on a 4.7 Tesla Magnex Scientific MR scanner controlled by Agilent Technologies 
VnmrJ 3.1 console software. A $38 \mathrm{~mm}$ quadrature transmit/receive radiofrequency coil tuned to $200.6 \mathrm{MHz}$ was used (Air MRI). Anesthesia was initially induced $<2 \%-3 \%$ isoflurane delivered in medical grade air $\left(70 \% \mathrm{~N}_{2} / 30 \% \mathrm{O}_{2}\right.$ ) for $30-60 \mathrm{~s}$ (flow rate $0.1 \mathrm{~L} / \mathrm{min}$ ), and the levels of isoflurane were then maintained between $1 \%$ and $1.2 \%$ throughout the entire setup and imaging. Mice were placed prone on a plastic cradle with a respiratory pillow connected to a force transducer placed underneath the abdomen. Body temperatures were maintained using a warm air recirculation system that received feedback from a fiber optic thermocouple probe (SA Instruments). Respiratory rates were monitored continuously, and average breaths per minute across subjects were $30-40$. Two sets of functional images were acquired using a two-shot $\mathrm{T}^{*}$ weighted EPI sequence with the following parameters: $\mathrm{TR}=1 \mathrm{~s}, \mathrm{TE}=16$ $\mathrm{ms}$, flip angle $=90^{\circ}$, data matrix $64 \times 64$, FOV $25.6 \times 25.6 \mathrm{~mm}(0.4 \mathrm{~mm}$ voxel size), 10 slices at $1.2 \mathrm{~mm}$ thick each, with a total of 180 repetitions (at $2 \mathrm{~s}$ each, for a total $6 \mathrm{~min}$ per EPI scan). The two sets of imaging time series were used to average these and increase signal-to-noise. Slices were in axial view (coronal in the mouse) with the first slice starting at the rostral-most extension of the prefrontal/motor cortex and excluding the olfactory bulb. Fast spin echo anatomical scans were collected using the following parameters: $\mathrm{TR}=2 \mathrm{~s}$, effective $\mathrm{TE}=48 \mathrm{~ms}$ (RARE factor $=$ 8 ; echo spacing $=12 \mathrm{~ms}), 10$ averages ( $11 \mathrm{~min}$ scan time). The optogenetic BLS pattern was $20 \mathrm{~ms}$ pulses, $15 \mathrm{~Hz}, 150$ pulses/sequence, 10 repeated sequences with $20 \mathrm{~s}$ delay for $5 \mathrm{~min}$.

MRI processing and statistical analysis. Brain masks were drawn manually over anatomical and functional scans using image segmentation tools in ITK-SNAP (www.itksnap.org). The whole-brain masks were used to crop images and remove nonbrain voxels. This step improves realignment of functional scans and anatomical scans to the segmented atlas of the mouse brain using the FMRIB Software Library (FSL) linear registration program FLIRT (Jenkinson et al., 2002). This was performed as previously reported (Colon-Perez et al., 2016). Displacements in individual images over the series of 180 images and slice timing delays were corrected, and time series spikes were removed using Analysis of Functional NeuroImages (AFNI) (Cox, 1996). Following these preprocessing steps, the two time series images were averaged before statistical analysis using fMRI Expert Analysis Tool (FEAT version 6.0) program in FSL. A high pass temporal filter was applied to remove slow signal drifts (filter cutoff at $60 \mathrm{~s}$ ). In FSL, functional data were convolved with a gamma variate function based on a time series block of 1020 s light OFF and 10 $10 \mathrm{~s}$ light $\mathrm{ON}$ blocks. Functional activation in response to BLS was assessed by statistically ( $t$ test) comparing on versus off blocks. Cluster thresholding in each subject map was used to correct BOLD activation below a significance threshold of $p=0.05$. Groups maps were then generated using a fixed-effects ANOVA contrasting activation versus no activation. FSL featquery was finally used to determine BOLD signal changes in voxel time series for PFC, NAc, BNST, and amygdala for each mouse. Time series plots were finally prepared using Prism version 7.0 (GraphPad).

In vitro electrophysiology. Mice received intraperitoneal injection of ketamine ( $0.1 \mathrm{ml}$ of $2 \%$ ketamine in sterile physiological saline) and were rapidly decapitated using a small-animal guillotine. Their brains were rapidly extracted, and coronal sections (300 $\mu \mathrm{m}$ thick) were made using a VT1000s vibratome (Leica Microsystems). During this procedure, tissue was submerged in ice-cold sucrose-laden oxygenated ACSF containing the following (in mM): 206 sucrose, 10 D-glucose, $1 \mathrm{MgSO}_{4} 2 \mathrm{KCl}$, $1.25 \mathrm{NaH}_{2} \mathrm{PO}_{4}, 1 \mathrm{CaCl}_{2}$, and $25 \mathrm{NaHCO}_{3}$. Slices were then incubated for $30 \mathrm{~min}$ at $35^{\circ} \mathrm{C}$ in a low-calcium ACSF containing the following (in mM): $124 \mathrm{NaCl}, 10$ D-glucose, $3 \mathrm{MgSO}_{4}, 2.5 \mathrm{KCl}, 1.23 \mathrm{NaH}_{2} \mathrm{PO}_{4}, 1 \mathrm{CaCl}_{2}$, and $25 \mathrm{NaHCO}_{3}$. All extracellular bath solutions were saturated with $95 \% \mathrm{O}_{2} / 5 \% \mathrm{CO}_{2}$ to maintain a $\mathrm{pH}$ of 7.3 . Following incubation, slices were permitted to passively equilibrate to room temperature $>30 \mathrm{~min}$ before recording.

In vitro imaging of GCaMP6f. GCaMP6f was imaged through a $4 \times$ lens at $\sim 3$ frames per minute. Average pixel intensity was calculated over time in multiple ROIs, each centered around an individual GCaMP6f-positive neuron (or small group of neurons) using FIJI (Schindelin et al., 2012). The average pixel intensity in each ROI over time was then normalized to a baseline period lasting at least $2 \mathrm{~min}$ and occurring immediately before bath application of oxytocin. These normalized fluorescence intensities were then averaged across all ROIs to produce the overall $\Delta \mathrm{F} / \mathrm{F}$ as observed in response to oxytocin and presented in Figure 1 (where shaded area represents the SEM).

Whole-cell recording and optogenetic stimulation. All experiments were performed in ACSF containing the following (in mM): $126 \mathrm{NaCl}, 11$ D-glucose, $1.5 \mathrm{MgSO}_{4}, 3 \mathrm{KCl}, 1.2 \mathrm{NaH}_{2} \mathrm{PO}_{4}, 2.4 \mathrm{CaCl}_{2}$, and $25 \mathrm{NaHCO}_{3}$, saturated with $95 \% \mathrm{O}_{2} / 5 \% \mathrm{CO}_{2}$, and maintained at $30^{\circ} \mathrm{C}-32^{\circ} \mathrm{C}$. Wholecell patch pipettes were pulled from borosilicate glass with a Flaming/ Brown P-97 micropipette puller (Sutter Instrument), and had an open tip resistance of 4-6 $\mathrm{M} \Omega$ when filled with an internal solution that contained the following (in mMM): $125 \mathrm{~K}$-gluconate, 10 phosphocreatine, 1 $\mathrm{MgCl}_{2}, 10 \mathrm{HEPES}, 0.1$ EGTA, $2 \mathrm{Na}_{2} \mathrm{ATP}$, and $0.25 \mathrm{Na}_{3} \mathrm{GTP}$. This solution was used for experiments that involved detailed intrinsic property characterization of BLA neurons in Figure 8. In most other experiments, a high chloride internal solution was used, which contained the following (in $\mathrm{mm}$ ): $100 \mathrm{~K}$-gluconate, $40 \mathrm{KCl}, 10 \mathrm{NaCl}, 10$ phosphocreatine, 2 $\mathrm{MgCl}_{2}, 10$ HEPES, 1 EGTA, $2 \mathrm{Na}_{2} \mathrm{ATP}$, and $0.3 \mathrm{Na} 3 \mathrm{GTP}$. This internal was considered preferable in cases where it was desirable for both excitatory and inhibitory synaptic currents to be strongly inward in neurons voltage-clamped at $-70 \mathrm{mV}$. Both solutions had an osmolarity of $\sim 295$ mOsm when adjusted to a $\mathrm{pH}$ of 7.25 with $\mathrm{KOH}$, and both solutions were passed through a $0.22 \mu \mathrm{m}$ filter before use. Before recording, neurons were visualized using a combination of differential interference contrast and epifluorescence microscopy on a BX51 WI upright microscope (Olympus) equipped with a 12-bit IR DIC camera, and a $40 \times$ waterimmersion objective. Epifluorescence imaging of eYFP-positive neurons was achieved using an X-Cite Series 120Q light source (Lumen Dynamics) and an appropriate filter set (XF404, excitation: 450-490 nm; emission: $510-560 \mathrm{~nm}$ ) from Omega Optical. Whole-cell patch-clamp recordings were made using a Multiclamp 700B amplifier (Molecular Devices), and data were collected at $20 \mathrm{kHz}$, lowpass filtered at $2 \mathrm{kHz}$, and recorded to disk using a Digidata 1440A digitizer controlled by Clampex version 10.2 (Molecular Devices). When necessary, channelrhodopsin2 (ChR2) or GCaMP6f was activated using a TTL-controlled blue LED $(\sim 488 \mathrm{~nm})$. For some experiments, the entire field was activated, whereas in others a fiber-coupled LED was used and light from the fiber was directed through a glass pipette. Individual neurons were discarded if access resistance (calculated from peak capacitive transients in the current response to repeated hyperpolarizing voltage steps) changed by $\sim>20 \%$ during the course of an experiment. Bath-applied pharmacological agents were used at the following concentrations (in $\mu \mathrm{M}$ ): 20 DNQX, 40 AP-5, 10 CGP 55845, 100 picrotoxin, and 0.2-0.4 oxytocin (OXT). All agents were purchased from Tocris Bioscience, except for picrotoxin, which was purchased from Sigma-Aldrich. All electrophysiological data were analyzed offline using custom software written in OriginC (OriginLab) by C.J.F.

Biocytin labeling. In experiments that involved post hoc evaluation of cell morphology, $5 \mathrm{~mm}$ biocytin was added to the internal solutions noted above. At the end of each experiment, the patch pipette was slowly withdrawn diagonally, and under visual control, using differential interference contrast microscopy. Slices were then transferred into $10 \%$ formalin at $4^{\circ} \mathrm{C}$ for $12-48 \mathrm{~h}$. Subsequent procedures were performed at room temperature on an orbital shaker. Slices were washed $(5 \times 5 \mathrm{~min}$ in PBS), and then permeabilized in PBST (PBS $+0.1 \%$ Triton X-100) supplemented with $2 \% \mathrm{BSA}$ and $0.1 \% \mathrm{NaN}_{3}$ for $48 \mathrm{~h}$. Fluorophore-conjugated streptavidin (Thermo Fisher Scientific, S32357 or S32356) was added (1:1000) for $24 \mathrm{~h}$. Following $5 \times 5 \mathrm{~min}$ washes in PBS, tissues were mounted on coverslips using an aqueous mounting media (Vectshield) and allowed to rest for $24 \mathrm{~h}$ before epifluorescence imaging.

Statistics. Data were analyzed and graphed using Prism 5 for Windows (GraphPad Software), OriginPro 2018 (Originlab), and STATISTICA 8.0 (StatSoft). While evaluating the effect of ChR2 on social behaviors, including social novelty test, the novel object recognition test, and the social interaction test, statistical significance was determined using a two-factor ANOVA with repeated measures. Stimulus (mouse vs object; novel vs familiar) or treatment (ChR2 vs control) were the two factors. Post hoc analysis was conducted using a Bonferroni post-test. When evaluating the effect of blue light, statistical significance was analyzed using a 
A

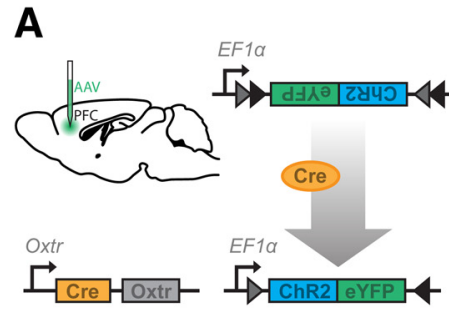

B

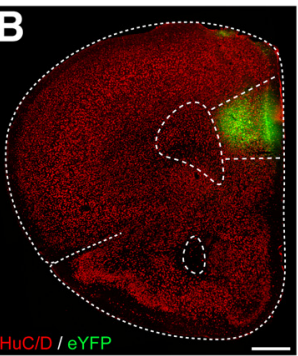

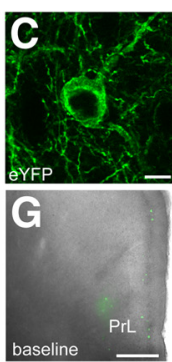
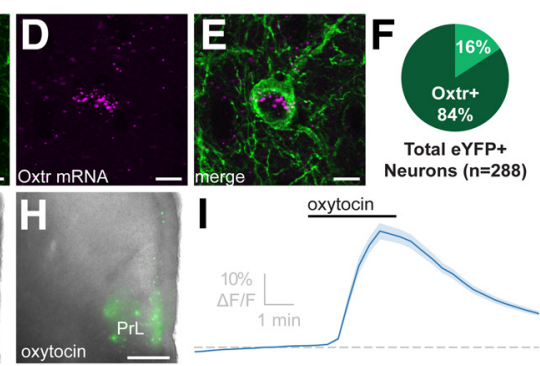

Figure 1. Assessing the direction of Cre-recombinase to Oxtr in the PFC. $A$, Schematic illustrating (left) bilateral injections of the AAV-ChR2-eYFP into the PFC of Oxtr-Cre mice, (right, top) the viral construct used to specifically target OXTR-expressing neurons, and (right, bottom) the Cre-mediated inversion of the eYFP and ChR2 sequence that results in the expression of these genes. $\boldsymbol{B}$, Unilateral coronal brain section through the PFC of an Oxtr-Cre mouse delivered AAV-ChR2-eYFP, demonstrating the specific transfection of PFC neurons (eYFP, green) relative to all neurons (HuC/D, red). C, A projection image of an eYFP-labeled (green) neuron residing in the PFC, (D) Oxtr mRNA (magenta), (E) merged image, and ( $\boldsymbol{F}$ ) pie graph showing the frequency by which eYFP-labeled PFC neurons also express Oxtr mRNA. G, Representative unilateral coronal section through the PFC demonstrating GCaMP6f fluorescent intensity before, and then $(\boldsymbol{H})$ after bath application of $200 \mathrm{~nm}$ oxytocin. I, Average change in GCaMP6f fluorescent intensity following application of $200 \mathrm{~nm}$ oxytocin. Scale bars: $\boldsymbol{B}, 500 \mu \mathrm{m} ; \boldsymbol{C}-\boldsymbol{E}, 10 \mu \mathrm{m} ; \boldsymbol{G}, \boldsymbol{H}, 100 \mu \mathrm{m}$.

three-factor ANOVA with repeated measures. The factors were stimulus (mouse vs object; novel vs familiar), treatment (ChR2 vs control), or light (no light vs BLS). Post hoc analysis was also used a Bonferroni post-test. Anxiety-like behaviors (including EPM test and Open Field Test) were evaluated using two-tailed Student's $t$ test (EPM) or two-way ANOVA with Bonferroni post-test (open field). A one-sample Student's $t$ test was used to evaluate the effect of oxytocin on GCaMP-positive OXTRexpressing cells in vitro (null hypothesis mean $=0$ ) and the effect of bath-applied antagonists on the normalized amplitude of light-evoked responses (null hypothesis mean $=1$ ).

\section{Results}

\section{Cre recombinase is produced by cells in the PFC that express} functional OXTRs

We obtained transgenic mice with Cre recombinase directed by the promoter for Oxtr to investigate the structure and function of neurons in the PFC that express OXTRs. To assess the fidelity of Cre direction to Oxtr, we bilaterally delivered Cre-inducible AAV-producing ChR2 and eYFP into the PFC of male Oxtr-Cre mice (Fig. 1A). This is expected to promote the expression of eYFP and ChR2 specifically in neurons in the PFC that synthesize Cre recombinase and OXTRs, thereby allowing visualization and optogenetic excitation of this discrete population of neurons. Figure $1 B$ depicts the location of virus-transfected neurons in a representative unilateral coronal brain section through the PFC. We found that Oxtr mRNA was present in $84 \%$ of eYFP-labeled PFC neurons that were surveyed (288 neurons sampled from 3 Oxtr-Cre mice; Fig. $1 C-F)$.

Next, we directed the expression of the ultrasensitive fluorescent protein calcium sensor, GCaMP6f (Dana et al., 2014), to cells that express Oxtr to assess whether application of exogenous oxytocin influenced neuronal activity as indicated by changes in intracellular free calcium. Cre-inducible AAV-expressing GCaMP6f was bilaterally delivered into the PFC of Oxtr-Cre mice, leading to the synthesis of GCaMP6f specifically in neurons within the PFC that express Oxtr. Two weeks after the injection, mice were killed and live brain slices through the PFC were used for in vitro experiments, evaluating the effect of bath application of oxytocin on GCaMP6f fluorescent intensity. Figure $1 G$ shows basal levels of GCaMP6f fluorescence (green) in an in vitro unilateral coronal preparation of the PFC. Subsequent application of $200 \mathrm{~nm}$ oxytocin dramatically increased GCaMP6f fluorescence intensity (Fig. $1 H$ ). Figure $1 I$ shows the significant effect that in vitro application of oxytocin had on GCaMP6f fluorescence measured in 220 ROIs sampled from 22 brain slices obtained from 4 Oxtr-Cre mice $\left(\Delta \mathrm{F} / \mathrm{F}=37.0 \pm 2.43 \%\right.$ of baseline, $t_{(219)}=15.23$, $p<1.0 \times 10^{-4}$ against null hypothesis of mean $=0$, one-sample
Student's $t$ test). Together, these results indicate high-fidelity direction of Cre recombinase to neurons in the PFC that express OXTRs, and further indicate that bath application of an exogenous OXTR agonist excites a subset of these OXTR-expressing neurons in vitro.

\section{OXTRs are expressed on glutamatergic and GABAergic neurons in the PFC}

Previous studies have used behavioral pharmacology to demonstrate the presence of functional OXTRs in the PFC (Sabihi et al., 2014); however, little is known about the phenotype of the cells expressing these receptors. Prior examination has focused exclusively on GABAergic interneurons (Nakajima et al., 2014; Li et al., 2016). To probe the neurochemical phenotype of OXTRexpressing neurons, Oxtr-Cre mice were administered Creinducible AAV-producing ChR2 and eYFP into the PFC. Two weeks later, mice were perfused and brains were processed for immunohistochemistry for eYFP and double-label RNAscope ISH for Gad1 and VGLuT1 mRNAs, which are markers for GABA or glutamate, respectively. Consistent with previous reports $(\mathrm{Na}-$ kajima et al., 2014; Li et al., 2016), many neurons labeled for eYFP were also positive for Gad1 mRNA, confirming that the OXTR is expressed on GABAergic neurons in the PFC (Fig. 2A-D). However, intriguingly, many eYFP neurons expressed VGluT1 mRNA rather than mRNA for Gad1 (Fig. 2E-H), suggesting that glutamatergic neurons in the PFC also express OXTR. In total, we sampled 288 neurons in the PFC from 3 Oxtr-Cre mice and found that 94 colabeled for Gad1 mRNA and eYFP. Additionally, 651 neurons in the PFC were sampled from 5 Oxtr-Cre mice, and 303 of these neurons colabeled for VGluT1 mRNA and eYFP. These neuroanatomical results suggest that a subset of both glutamatergic and GABAergic neurons in the PFC express OXTR.

To the best of our knowledge, the presence of OXTR on glutamatergic neurons in the PFC has not previously been reported. In an attempt to confirm or refute our neuroanatomical results, we conducted a series of in vitro electrophysiological studies to evaluate the membrane properties, firing behavior, morphology, and transmitter phenotype of OXTR-expressing neurons in the PFC. Toward this end, Oxtr-Cre mice were again administered Cre-inducible AAV-producing eYFP into the PFC; and then $\sim 2$ weeks later, whole-cell patch-clamp recordings were made from eYFP-labeled neurons to evaluate intrinsic properties of the cells, and to determine whether they were responsive to bath application of oxytocin. In total, we recorded from 39 eYFP-labeled neurons in the PFC obtained from 4 animals and found that a strong subset were clearly responsive to bath application of 200 
nM oxytocin as indicated by a decrease in rheobase observed in current clamp $\left(-17.7 \pm 5.25 \mathrm{pA}, n=9, t_{(8)}=3.37, p=\right.$ 0.01 , null hypothesis mean $=0$, onesample $t$ test) or by a change in holding current observed in cells voltage-clamped at $-50 \mathrm{mV}\left(-28.3 \pm 8.5 \mathrm{pA}, n=5, t_{(4)}=\right.$ 3.2, $p=0.03$, null hypothesis mean $=0$, one-sample $t$ test). Consistent with previous reports (Nakajima et al., 2014; Li et al., 2016), some oxytocin-responsive neurons $(n=5)$ were categorized as interneurons due to lack of a primary apical dendrite, and a combination of small whole-cell capacitance $(42.9 \pm 12.0 \mathrm{pF})$, fast repolarization velocity $(85.6 \pm 8.8 \mathrm{mV} / \mathrm{ms})$, and/or a higher sustained firing rate in response to a $300 \mathrm{pA}$ current injection $(34.5 \pm 9.4 \mathrm{~Hz})$. A representative cell from this category and its response to bath application of $200 \mathrm{~nm}$ oxytocin are illustrated in Figure 2I, J. Interestingly, careful analysis of intrinsic properties and morphology of the remaining oxytocinresponsive eYFP-labeled neurons indicated that many of them were indeed pyramidal-like. These cells were defined by a soma located in layer $2 / 3$ or layer $5 / 6$, the presence of a single primary spiny api-

cal dendrite, an average whole-cell capacitance of $81.32 \pm 13.04$ $\mathrm{pF}(n=9)$, a repolarization velocity of $48.76 \pm 2.03 \mathrm{mV} / \mathrm{ms}(n=$ 9 ), and a maximum firing rate of $17.77 \pm 2.54 \mathrm{~Hz}(n=9)$ (as observed in response to $300 \mathrm{pA}$ of current injection). A representative cell in this category and its response to bath application of $200 \mathrm{~nm}$ oxytocin are illustrated in Figure $2 K, L$. Overall, these electrophysiological data reinforce the conclusion that functional OXTRs are expressed on both pyramidal cells and interneurons in the PFC.

Subsequent experiments used in vitro optogenetics to determine whether neurons in the PFC that express OXTR release GABA or glutamate onto other PFC neurons. Again, Oxtr-Cre mice were given Cre-inducible AAV-eYFP-ChR2 into the PFC, killed 10-14 d later, and whole-cell patch-clamp recordings were obtained from PFC neurons in vitro. BLS of ChR2/eYFPexpressing neurons reliably induced action potentials in current clamp, indicating that optogenetic stimulation robustly excites OXTR-expressing neurons in the PFC (Fig. 3A, bottom). Next, voltage-clamp recordings obtained from neighboring neurons devoid of eYFP and ChR2 revealed that BLS elicited two types of postsynaptic currents in the PFC: some that were abolished by bath application of ionotropic glutamate receptor antagonists (DNQX, AP5, Fig. 3B, middle, bottom; $t_{(7)}=258.15 ; p<1 \times$ $10^{-4}$, null hypothesis mean $=1$, one-sample Student's $t$ test), and others that were abolished by GABA receptor antagonists (PTX, CGP, Fig. 3C, middle, bottom; $t_{(5)}=33.38 ; p<1 \times 10^{-4}$, null hypothesis mean $=1$, one-sample Student's $t$ test). In one neuron, BLS elicited postsynaptic currents carried by both glutamate and GABA, indicating that a single PFC neuron may receive input from both glutamatergic and GABAergic OXTRexpressing PFC neurons (Fig. 3D). Collectively, these results demonstrate that glutamatergic pyramidal neurons and GABAergic interneurons in the PFC express functional OXTRs.
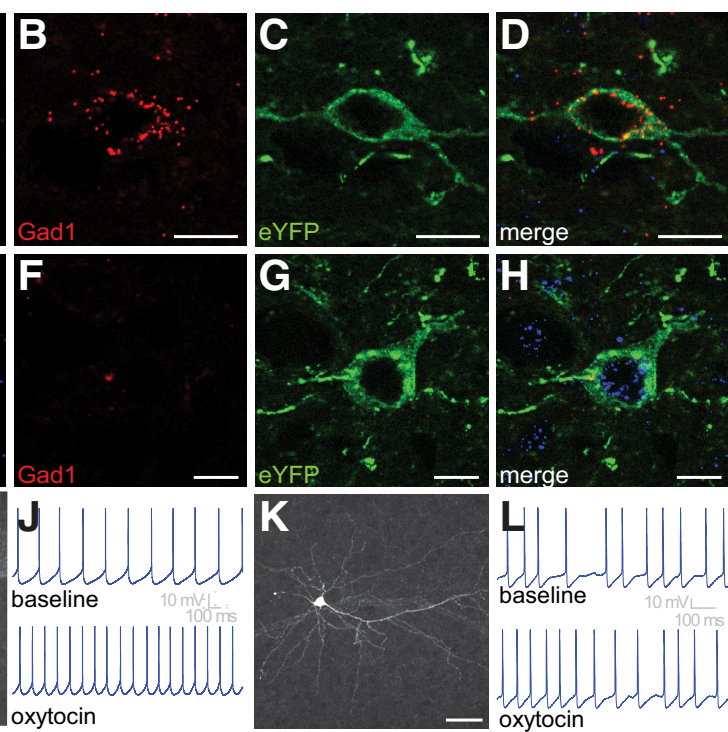

oxytocin

Figure 2. Neurochemical and electrophysiological phenotypes of OXTR-expressing neurons in the PFC. $\boldsymbol{A}-\boldsymbol{H}$, Representative

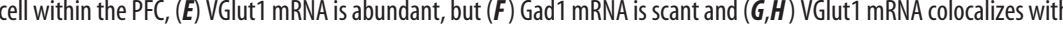
Pyramidal-like eYFP-labeled neurons in the PFC are also excited by bath application of $200 \mathrm{~nm}$ oxytocin (example recorded under constant 300 pA current injection). Scale bars: $\boldsymbol{A}-\boldsymbol{H}, 10 \mu \mathrm{m} ; \boldsymbol{I}, \boldsymbol{K}, 50 \mu \mathrm{m}$.

In vivo optogenetic activation of OXTR-expressing neurons in the PFC excites subcortical nuclei

Next, we combined the use of in vivo optogenetics with fMRI to discern whether excitation of OXTR-expressing neurons in the PFC affects neuronal activity within subcortical structures. Creinducible AAV-eYFP-ChR2 was bilaterally injected into the PFC of Oxtr-Cre mice $(n=8)$, and chronic dwelling fiber optics targeting the PFC were implanted (Fig. 4A). Three weeks after implantation, mice were lightly anesthetized with $1 \%$ isoflurane using a protocol previously shown to allow measurable BOLD responses (Bukhari et al., 2018). Subsequently, mice were tethered to fiber optic cables connected to a $473 \mathrm{~nm}$ laser light source and placed in a $4.7 \mathrm{~T}$ scanner. As anticipated, fMRI showed that BLS significantly $(p<0.05)$ increased BOLD activation in the PFC, with activation in the prelimbic cortex being particularly robust. Importantly, however, increased BOLD activation was also observed in several subcortical structures, including the amygdala, BNST, and NAc ( $p<0.05$; Fig. $4 B, C)$. Of these regions, the BNST and amygdala showed somewhat variable but significant activation compared with that of the NAc, which showed somewhat weaker, albeit still significant, activation (Fig. $4 D)$. Overall, these results indicate that BLS delivered to ChR2transfected PFC neurons elicits significant neuronal activation in vivo, and further suggest that activation of OXTR-expressing neurons in the PFC is temporally coupled to downstream activation of brain nuclei implicated in anxiety, reward, and social behaviors.

\section{OXTR-expressing neurons in the PFC send robust} glutamatergic projections to mesolimbic nuclei

Follow-up neuroanatomical experiments tested the hypothesis that the increased BOLD activation observed in mesolimbic nuclei resulted from activation of direct excitatory inputs originating from OXTR-expressing neurons in the PFC. Cre-inducible AAV-ChR2-eYFP was bilaterally delivered into the PFC of Oxtr- 

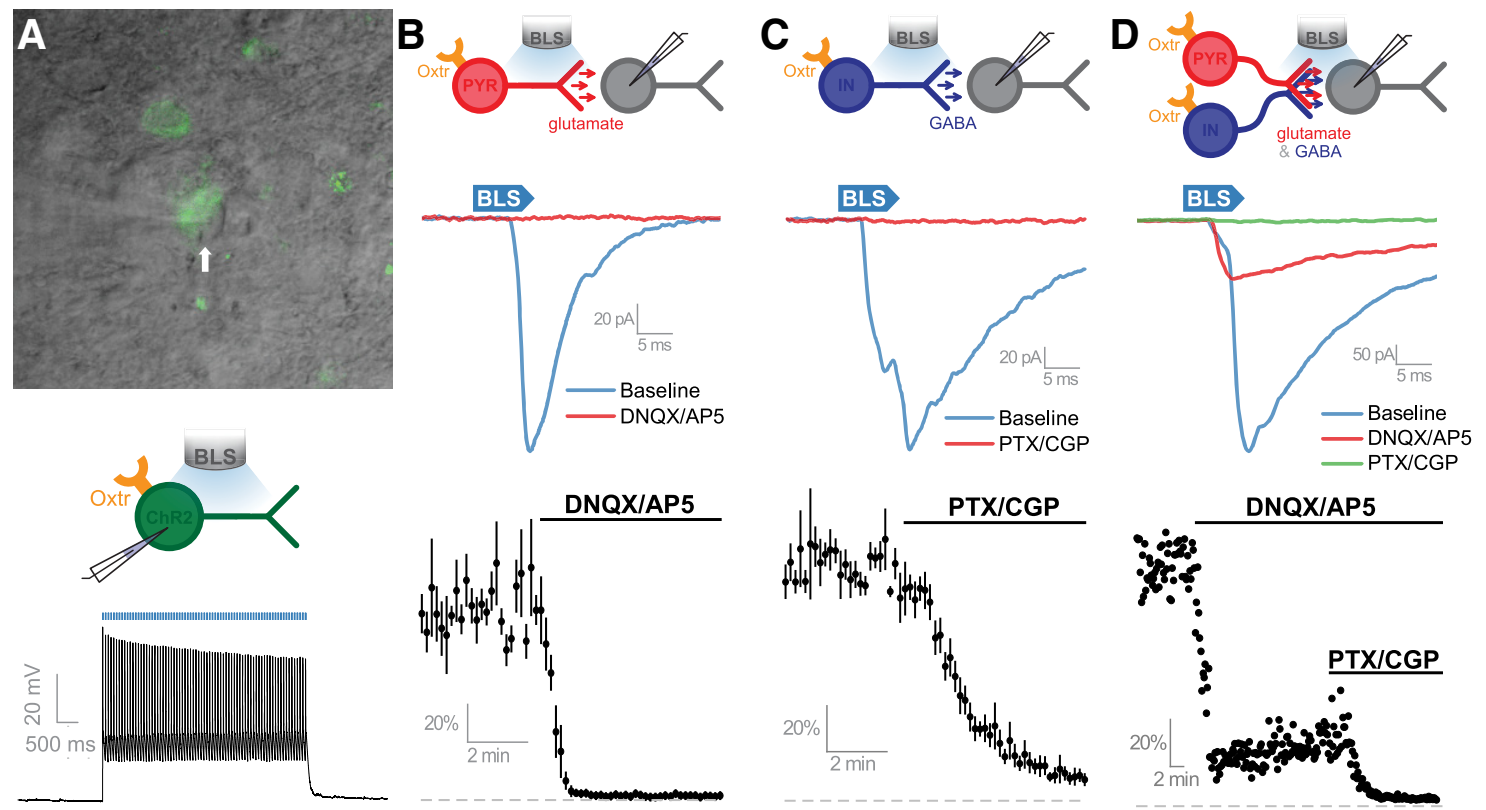

Figure 3. OXTR-expressing neurons release glutamate and GABA onto neighboring neurons in the PFC. $A$, Top, Viral delivery of eYFP and ChR2 into OXTR-expressing neurons allows their identification with epifluorescence and DIC microscopy. Middle, Schematic of patch-clamp recordings obtained from OXTR-expressing neurons transfected with ChR2 that were exposed to blue light stimulation (BLS). Bottom, A pulse train of BLS reliably causes the generation of action potentials in 0XTR-expressing neurons in the PFC that were transfected with ChR2 (BLS, $20 \mathrm{~ms} \mathrm{pulses,} 15 \mathrm{~Hz}$ ). B, Top, Schematic of patch-clamp recordings taken from PFC postsynaptic neurons identified as receiving synaptic inputs from eYPF/ChR2-positive 0XTR-expressing glutamatergic neurons in the PFC. Middle, In some eYFP/ChR2-negative PFC pyramidal-like neurons, BLS evoked synaptic currents (blue trace) that are blocked by bath application of ionotropic glutamate receptor antagonists (red trace). Bottom, Average normalized response to BLS from all cells studied that had evoked responses sensitive to glutamate receptor antagonists DNQX and AP5. C, Top, Schematic of patch-clamp recordings taken from PFC postsynaptic neurons identified as receiving GABAergic inputs by BLS of OXTR-expressing neurons. Middle, BLS evokes synaptic currents in conditions that isolate GABAergic signaling. Bottom, Average normalized light-evoked GABA current of all cells studied demonstrating sensitivity to GABA receptor antagonists PTX and CGP. Scale bar: $A, 20 \mu \mathrm{m}$. $D$, Top, Schematic depicting that OXTRs are expressed by pyramidal or interneurons with BLS eliciting glutamate and GABA release onto the same postsynaptic neuron. Middle, Representative responses to BLS. Bottom, Normalized light-evoked response was partially DNQX/AP5 sensitive and fully eliminated by PTX/CGP.

Cre mice as previously described. Ten to 14 days later, brains were extracted and used for neuronal tract-tracing studies. Viral transfection of OXTR-expressing neurons in the PFC results in robust expression of eYFP-labeled axons that appear to terminate in the NAc, BNST, and BLA (Fig. 5A, top, bottom). In particular, eYFPlabeled axons nearly encapsulated the entire anterior portion of the BLA (bregma -1.06 to -1.82 ) (Franklin and Paxinos, 2008). Additional eYFP-labeled axons were observed in midbrain regions, including the VTA and dorsal raphe nucleus, regions that also exhibited increased BOLD signal with in vivo optogenetic activation of the PFC ( $p<0.05$; data not shown). To evaluate whether the eYFP-labeled axons that were observed in the forebrain were fibers of passage or nerve terminals, Oxtr-Cre mice had Cre-inducible AAV-eYFP-ChR2 delivered into the PFC and the retrograde tracer, FG, was iontophoretically applied into the NAc, BNST, or BLA. The resulting immunohistochemistry on brain sections through the PFC revealed that eYFP-labeled neurons were also labeled with $\mathrm{FG}$, indicating that axons terminating in the NAc, BNST, or BLA originate from the somas of OXTRexpressing neurons residing within the PFC (Fig. $5 B$ ).

Next, we used in vitro electrophysiological tools to confirm that OXTR-expressing neurons in the PFC form functional synaptic terminals in the NAc, BNST, and BLA, and further to identify the transmitter released from these terminals. Toward that end, another group of Oxtr-Cre mice were administered AAVeYFP-ChR2 into the PFC; and 2-3 weeks later, brain slices through the NAc, BNST, or BLA were used for CRACM. Neurons in these areas were voltage-clamped at $-70 \mathrm{mV}$ using an internal solution that contained a high concentration of chloride, and BLS was used to evoke transmitter release from axon terminals arising from OXTR-expressing neurons in the PFC (Fig. 6A-C, middle). In each area tested, BLS evoked clear inward currents that were completely blocked by bath application of ionotropic glutamate receptor antagonists (Fig. $6 A-C$, for NAc, BNST, and BLA, respectively: $n=9, t_{(8)}=49.07, p<1 \times 10^{-4}, n=3, t_{(2)}=51.96$, $p=1 \times 10^{-4}$, and $n=9, t_{(8)}=26.55, p<1 \times 10^{-4}$, null hypothesis mean $=1$, one-sample Student's $t$ test). By contrast, these experiments did not reveal any evidence of long-range GABAergic projections. Together, these results suggest that neurons in the PFC that express OXTR have robust glutamatergic projections to the NAc, BNST, and BLA that may underlie the increased BOLD activation that was observed in these brain nuclei during in vivo optogenetic activation of OXTR-expressing PFC neurons.

\section{In vivo optogenetic activation of OXTR-expressing neurons in the PFC impairs social recognition}

The connectivity and neurochemical phenotype of OXTRexpressing neurons in the PFC predict top-down excitatory influence over mesolimbic brain nuclei implicated in social, rewarding, and anxiety-like behaviors. To assess whether the activity of OXTR-expressing neurons in the PFC modulates these behaviors, Oxtr-Cre mice were injected with Cre-inducible AAVeYFP (Control) or AAV-ChR2-eYFP (ChR2) as previously described but were subsequently bilaterally implanted with chronic dwelling fiber optics targeting the PFC. Figure $7 A$ is a representative coronal section through the PFC depicting the juxtaposition of virally transfected neurons and chronically implanted fiber optics, thereby demonstrating the high probability of BLS affecting neurons residing within the region of interest. After 
A

B

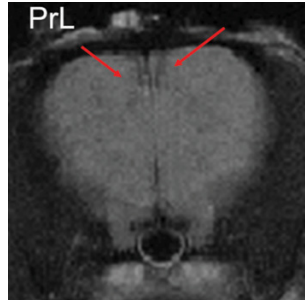

C

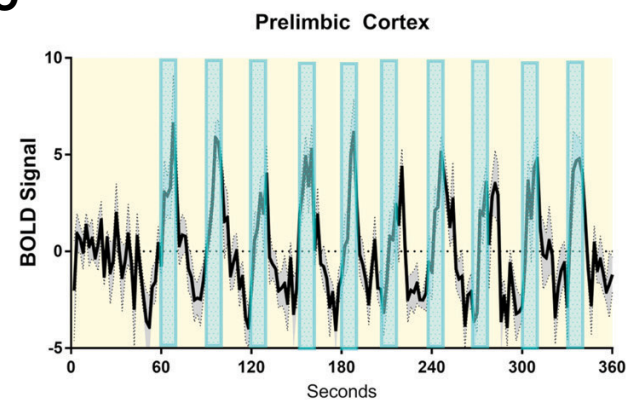

BNST

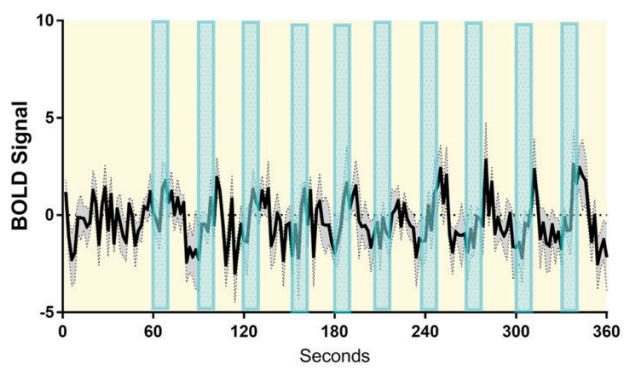

\section{PrL}

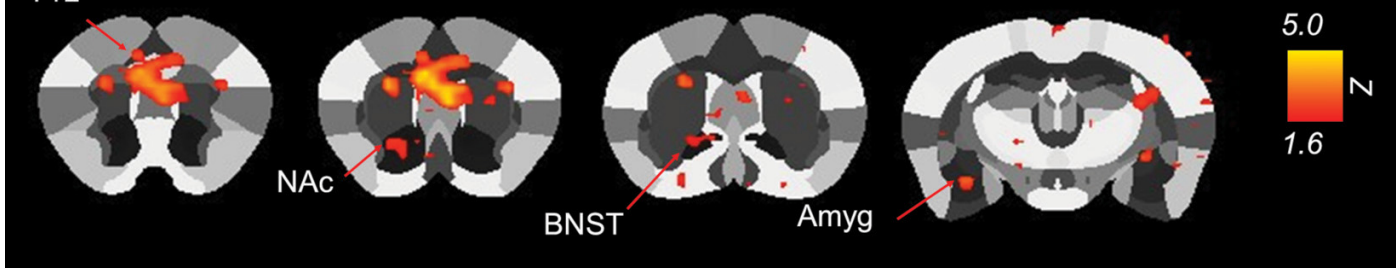

Amygdala

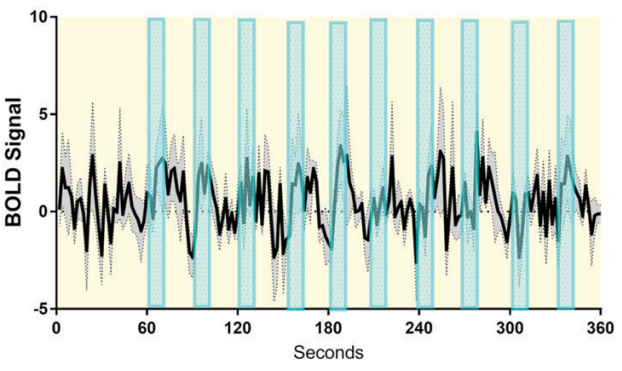

Nucleus Accumbens

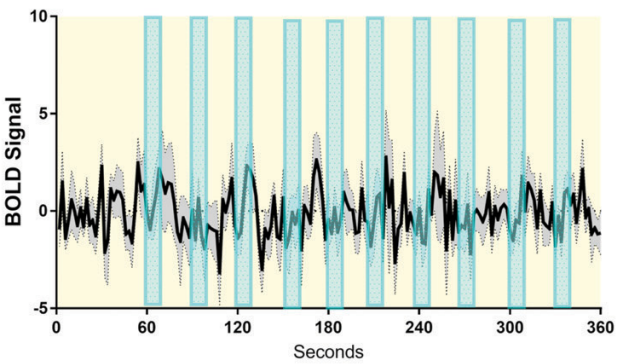

D

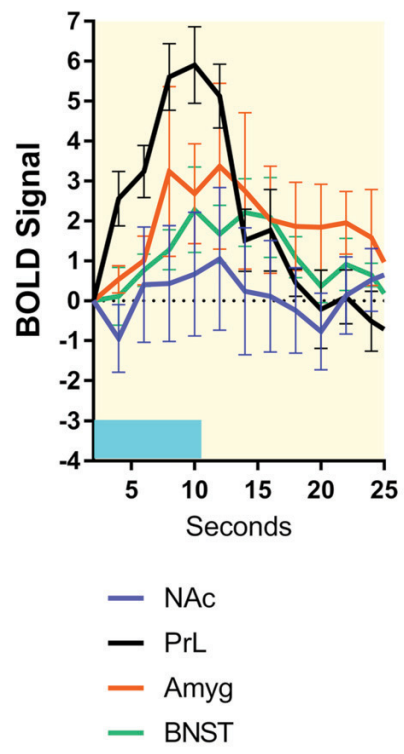

Figure 4. Optogenetic activation of OXTR-expressing neurons in the PFC produces excitatory responses in multiple brain areas as revealed by fMRI. $\boldsymbol{A}$, Representative coronal scan through the mouse PFC depicting the placement of chronic dwelling fiber optic implants just dorsal to the PFC. $\boldsymbol{B}$, BOLD activation maps aligned and overlaid onto a segmented atlas of the mouse brain. Maps were cluster-thresholded $(p<0.05)$. Arrows indicate areas that had significantly activated voxels. $C$, Time series BOLD responses to light stimulation in the PrL, amygdala, NAC, and BNST. $\boldsymbol{D}$, Averaged BOLD waveforms during the window of $\boldsymbol{B}$. Amyg, Amygdala; PrL, prelimbic cortex.

surgical recovery, we first assessed whether excitation of OXTRexpressing neurons in the PFC altered sociability, as defined as the amount of time the subject mouse spends with the novel conspecific or an inanimate novel object when placed in the three-chambered social test (Fig. $7 B$, top). We found that there was an effect of stimulus $\left(F_{(1,8)}=104.10 ; p<1.0 \times 10^{-4}\right)$ with both control mice and those expressing ChR2 spending significantly more time exploring the novel conspecific than the object (control: $n=5, p=6.5 \times 10^{-4}$; ChR2: $n=5, p=4.6 \times 10^{-4}$ ). However, no effect of treatment $\left(F_{(1,8)}=4.02 ; p=0.08\right)$ or stimulus $\times$ treatment interaction $\left(F_{(1,8)}=0.059 ; p=0.81\right)$ was observed (Fig. $7 B$, bottom). Next, we evaluated whether excitation of OXTR-expressing neurons in the PFC affected social memory as indicated by preference for novel conspecifics in the social recognition test (Fig. $7 C$, top). As expected, control mice exhibited a clear preference for novel conspecifics (Fig. 7C, bottom, left). There was a stimulus $\times$ treatment interaction $\left(F_{(1,9)}=8.11\right.$, $p=0.02)$, and post hoc analysis revealed that control mice spent significantly (control $n=5, p=2.5 \times 10^{-3}$ ) more time exploring the novel conspecific. In contrast, optogenetic excitation of neurons in the PFC that express OXTRs abolished this preference and caused mice to spend similar amounts of time investigating conspecifics that were novel or familiar (Fig. 7C, bottom, right; ChR2 $n=6, p=0.70$ ). We repeated this experiment in another cohort of mice to control for the possibility that viral expression of ChR2 impairs social recognition independent of BLS-induced neuronal activation; however, in the absence of BLS, a significant preference for novel conspecifics was observed in mice treated with AAV-eYFP or AAV-eYFP-ChR2. Specifically, there was an effect of stimulus $\left(F_{(1,20)}=73.78, p<1.0 \times 10^{-4}\right)$ with mice spending more time interacting with the novel conspecific. There was also a stimulus $\times$ treatment interaction $\left(F_{(1,20)}=9.80 ; p=5.3 \times\right.$ $\left.10^{-3}\right)$. However, post hoc analysis revealed that control mice without BLS stimulation (Control-NL, $n=5$ ), control mice with BLS (Control-BLS, $n=5$ ), and ChR2 mice without BLS stimulation (ChR2-NL, $n=8$ ) all exhibited a clear preference for social novelty as indicated by spending significantly more time exploring a novel conspecific (Control-NL: $58.43 \pm 8.40 \mathrm{~s}$ vs $22.49 \pm 3.59 \mathrm{~s}, p=1.8 \times 10^{-4}$; Control-BLS: $54.67 \pm 6.77 \mathrm{~s}$ vs $26.05 \pm 5.08 \mathrm{~s}, p=2.8 \times 10^{-3}$ and ChR2-NL: $48.38 \pm 4.59 \mathrm{~s}$ vs $26.64 \pm 5.07 \mathrm{~s}, p=4.4 \times 10^{-3}$, three-factor ANOVA with repeated measures). However, optogenetic excitation of OXTRexpressing neurons in the PFC abolished this effect, with ChR2 mice spending similar amounts of time exploring novel and familiar conspecifics (ChR2-BLS: $n=6,34.50 \pm 2.87$ s vs $26.16 \pm$ $4.40 \mathrm{~s}, p=1.00$, three-factor ANOVA with repeated measures). This suggests that excitation of OXTR-expressing neurons in the PFC is necessary to eliminate this preference.

Additional experiments used the novel object recognition paradigm (Fig. 7D, top) to determine whether activation of 


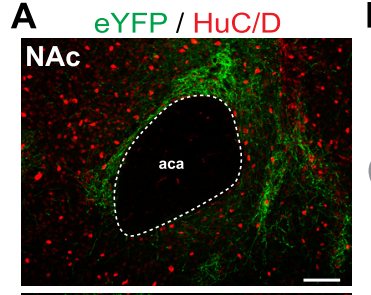

B
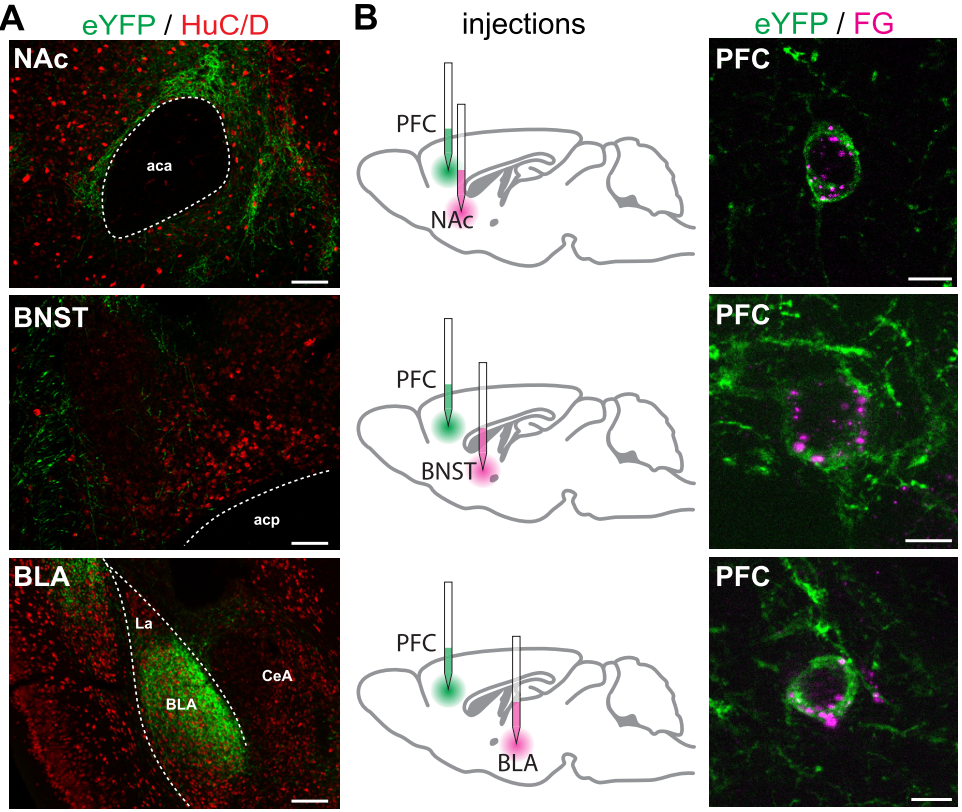

Figure 5. Anterograde and retrograde neuronal tract-tracing confirms that OXTR-expressing neurons in the PFC have axons that terminate in the NAC, BNST, and BLA. A, Representative photomicrographs depicting eYFP-labeled axons (green) originating from PFC OXTR-expressing neurons that appear to form appositions onto neurons (HuC/D; red) in the NAC (top), BNST (middle), and BLA (bottom). $\boldsymbol{B}$, Cre-dependent eYFP was virally delivered into the PFC in combination with iontophoretic delivery of the retrograde tracer FG into the NAc (top), BNST (middle), or BLA (bottom). Neurons colabeled for eYFP (green) and FG (magenta) represent OXTR-expressing neurons in the PFC with direct axonal projections to the brain nuclei injected with FG. Scale bars: $\boldsymbol{A}$, top, middle, $100 \mu \mathrm{m} ; \boldsymbol{A}$, bottom, $200 \mu \mathrm{m} ; \boldsymbol{B}, 10 \mu \mathrm{m}$. aca; Anterior commissure; acp, posterior portion of the anterior commissure; $L A$, lateral amygdaloid nucleus; $C$ A, central nucleus of the amygdala.

OXTR-expressing neurons in the PFC affected social recognition by causing generalized memory deficits and/or decreased propensity for novel exploration. As expected, control mice spent significantly more time exploring objects that were novel relative to those that were familiar; and remarkably, mice treated with AAV-eYFP-ChR2 performed similarly (Fig. 7D, bottom). There was an effect of stimulus $\left(F_{(1,12)}=125.17\right.$; control mice: $n=7$, $p<1.0 \times 10^{-4}$; ChR2 mice: $\left.n=7, p<1.0 \times 10^{-4}\right)$. However, no effect of treatment $\left(F_{(1,12)}=1.56 ; p=0.24\right)$ or stimulus $\times$ treatment interaction $\left(F_{(1,12)}=0.21 ; p=0.65\right)$ was observed. These results suggest that eliminating the preference for social novelty with optogenetic activation of OXTR-expressing neurons in the PFC is not the result of generalized memory deficits or aversion to novelty but, rather, may specifically impair social recognition. Subsequent experiments further tested this hypothesis by evaluating mice in the EPM and open field to determine whether the impaired social recognition that occurred with optogenetic activation of the PFC was the result of altered anxiety-like behavior or locomotor activity. Surprisingly, mice administered AAV$\operatorname{eYFP}(n=9$ or 10$)$ and those given AAV-ChR2-eYFP $(n=10)$ behaved similarly in the EPM, as shown by time spent in open arm (Fig. $\left.7 E ; \mathrm{df}=18, t=2.6 \times 10^{-3}, p=0.99\right)$, open arm entries (7F; $\mathrm{df}=18, t=0.86, p=0.4)$, closed arm entries (7G; $\mathrm{df}=17$, $t=1.42, p=0.17)$, and total distance traveled $(7 \mathrm{H} ; \mathrm{df}=18, t=$ $0.85, p=0.4)$. The Open Field Test also indicated that neither $\operatorname{BLS}\left(F_{(1,28)}=1.41, p=0.24\right)$ nor ChR2 transfection $\left(F_{(1,28)}=\right.$ 2.68, $p=0.11)$ affected anxiety-like behavior, as shown by the percentage of time that mice spent in the center of an open field (Control-NL: $12.2 \pm 1.85 \%, n=7$; Control-BLS: $11.82 \pm 1.47 \%$, $n=9$; ChR2-NL: $16.17 \pm 1.42 \%, n=7$; Control-BLS: $12.89 \pm$
$1.39 \%, n=9$ ). BLS in the PFC slightly increased locomotor activity (distance traveled $)$ in mice $\left(F_{(1,28)}=5.24 ; p=\right.$ $0.03)$; however, there was no stimulus $X$ treatment interaction $\left(F_{(1,28)}=0.5, p=\right.$ 0.49; Control-NL: $16,048 \pm 1223 \mathrm{~mm}$; Control-BLS: 17,702 $\pm 963.4 \mathrm{~mm}$; ChR2NL: $17,435 \pm 964.7 \mathrm{~mm}$; Control-BLS: $20,567 \pm 1002 \mathrm{~mm})$. Collectively, these results suggest that selectively activating OXTR-expressing neurons in the PFC impairs the ability to differentiate between novel and familiar conspecifics; however, this impairment appears to be a highly specific effect.

\section{Neurons in the PFC that are excited by oxytocin make glutamatergic synapses onto both pyramidal neurons and interneurons residing in the BLA}

Prior studies suggest that amygdala neurons, especially those located in the BLA, are important for coordinating brain activity when social interactions are initiated, and this may be critical for social recognition (Garrido Zinn et al., 2016; Tanimizu et al., 2017). The present study found that optogenetic activation of OXTR-expressing neurons in the PFC is associated with increased neuronal activity in the BLA and impaired social recognition. To gain insight on how this PFC to BLA pathway may control social recognition, we conducted two additional experiments using a combination of electrophysiological, neuroanatomical, and optogenetic tools. For the first series of experiments, retrograde tracer (fluorescent microspheres) was injected into the BLA of WT mice (Fig. $8 A$ ); and 48-72 h later, mice were killed and electrophysiological recordings were obtained from $\mathrm{PFC}$ neurons retrogradely labeled from the BLA. These neurons had intrinsic electrophysiological properties very similar to the eYFP-labeled pyramidal-like neurons in the PFC that were responsive to oxytocin (Fig. $2 K, L$ ). Specifically, average values for whole-cell capacitance, repolarization velocity, and maximum firing rate in response to a $300 \mathrm{pA}$ current injection were $89.5 \pm 5.45 \mathrm{pF}, 42.2 \pm 1.68 \mathrm{mV} / \mathrm{ms}$, and $16.6 \pm 0.82 \mathrm{~Hz}(n=53, n=48$, and $n=52$, respectively). To determine whether BLA-projecting PFC neurons in these WT animals were sensitive to oxytocin, we voltage-clamped the cells at $-50 \mathrm{mV}$ and then bath-applied 200-400 nM oxytocin. Across 53 neurons tested in this manner, oxytocin produced an average change in holding current of $-9.1 \pm 2.2 \mathrm{pA}$, as illustrated in Figure $8 E$. Twenty-six of these 53 neurons were considered responsive, as indicated by an absolute change in holding current $\geq$ $5 \mathrm{pA}$. The mean change in holding current in these responsive cells was $-19.1 \pm 3.2 \mathrm{pA}$ (not illustrated). Overall, these experiments indicate that BLA-projecting PFC neurons in WT mice have intrinsic properties consistent with being pyramidal neurons and are often excited by bath application of oxytocin.

For the second series of experiments, we used Oxtr-Cre mice that had received injection of AAV-ChR2-eYFP into the PFC. Two to 3 weeks after injection, we made acute tissue slices containing the BLA and used BLS to identify BLA neurons that receive excitatory input from the PFC. While this approach is 
reminiscent of that used in Figure 6, for this series of experiments, we used a K-glu-based internal with a lower concentration of chloride, intentionally targeted a wider range of BLA cell types, and performed a more extensive intrinsic property analysis of cells that received excitatory inputs in response to BLS. BLA neurons that responded to optogenetic stimulation were divided into two groups based on their whole-cell capacitance. Those that had whole-cell capacitance $<50 \mathrm{pF}($ mean $=22.0 \pm 2.8 \mathrm{pF})$ fired at $>20 \mathrm{~Hz}$ in response to a $300 \mathrm{pA}$ current injection $($ mean $=30.6 \pm 3.9 \mathrm{~Hz}, n=6$, representative response in Fig. $8 G$ ), whereas those that had whole-cell capacitance $>50 \mathrm{pF}(108 \pm 18.6 \mathrm{pF})$ fired significantly slower $($ mean $=16.4 \pm 1.9 \mathrm{~Hz}$, $n=6, p=0.008$ vs smaller cells, representative response in Fig. $8 H$ ). Figure $8 I$ further illustrates that whole-cell capacitance of BLS responsive BLA neurons was largely predictive of their firing rate. Although these groups did not similarly separate based on repolarization velocity or input resistance (not illustrated), we believe these results indicate that OXTR-expressing neurons in the PFC are likely to provide excitatory input to both interneurons and pyramidal neurons in the BLA.

\section{Selective optogenetic activation of an oxytocin-sensitive PFC to BLA circuit impairs social recognition}

To test the hypothesis that the impaired social recognition that we previously observed could be recapitulated by selectively stimulating the PFC to BLA projection, Oxtr-Cre mice were administered AAV-eYFP or AAV-ChR2-eYFP into the PFC and were bilaterally implanted with chronic dwelling fiber optics targeting the BLA (Fig. 9A, inset). Figure $9 A$ shows the representative site of injection and termination of the fiber optic slightly dorsal to eYFP-labeled axons in the BLA. Similar to our previous results, excitation of OXTR-expressing neurons in the PFC had no effect on sociability in the three-chambered social test (Fig. $9 B$, top). There was a significant effect of stimulus $\left(F_{(1,11)}=126.06 ; p<\right.$ $1.0 \times 10^{-4}$ ) with control mice and those expressing ChR2 spending significantly more time exploring the novel conspecific than the object (control: $n=6, p=1.4 \times 10^{-4}$; ChR2: $n=7, p<$ $\left.1.0 \times 10^{-4}\right)$. However, there was an effect of treatment $\left(F_{(1,11)}=\right.$ $5.41 ; p=0.04)$ but no stimulus $\times$ treatment interaction $\left(F_{(1,11)}=\right.$ $0.97 ; p=0.35)$. That is, control mice given AAV-eYFP and those given AAV-ChR2-eYFP preferentially investigated the novel conspecific relative to the object (Fig. $9 B$, bottom). In the social recognition test (Fig. 9C, top), there was a stimulus $\times$ treatment interaction $\left(F_{(1,10)}=5.37, p=0.04\right)$, with control mice exhibiting a clear preference for social novelty as indicated by spending significantly ( $p=0.04$ ) more time exploring a novel conspecific when BLS was delivered to the BLA. However, consistent with our hypothesis, this preference was completely eliminated by optogenetic stimulation of axons in the BLA arising from OXTRexpressing neurons in the PFC. That is, ChR2 mice spent similar $(p=1.00)$ amounts of time exploring novel and familiar conspe- cifics (Fig. 9C, bottom; control $n=6$, ChR2 $n=6$ ). Next, mice were evaluated in the novel object recognition test (Fig. 9D, top) to determine whether the effect on social recognition resulted from general memory impairments or aversion to novel exploration. Similar to what was observed when BLS was delivered to the PFC, mice treated with AAV-eYFP or AAV-ChR2-eYFP and given BLS into the BLA spent more time exploring the novel object relative to the familiar object (Fig. 9D, bottom). There was an effect of stimulus $\left(F_{(1,13)}=68.96 ; p<1.0 \times 10^{-4}\right)$ with control mice and those expressing ChR2 spending significantly (control: $n=8, p=3.4 \times$ $10^{-4}$; ChR2: $n=7, p=3.2 \times 10^{-4}$ ) more time exploring the novel object than the familiar object. However, no effect of treatment $\left(F_{(1,13)}=1.06 ; p=0.32\right)$ or stimulus $\times$ treatment interaction $\left(F_{(1,13)}=0.092 ; p=0.77\right)$ was observed.

To investigate whether optogenetic activation of this oxytocin-sensitive PFC to BLA circuit affected anxiety-like behavior and/or locomotor activity, mice were tested in the EPM and open field. Control mice $(n=11)$ and those given AAVChR2-eYFP ( $n=8$ or 9 ) had similar numbers of entries into the open arms of the EPM and spent similar amounts of time in the open arms of the EPM (Fig. $9 E$; $\mathrm{df}=18, t=0.29, p=0.78$ ) and had similar numbers of open arm entries (Fig. $9 F$; df $=18, t=$ $0.74, p=0.47$ ). However, mice given AAV-ChR2-eYFP entered the closed arms of the EPM significantly more often (Fig. 9G; $\mathrm{df}=17, t=2.52, p=0.02)$ and traveled a significantly further distance in the EPM (Fig. $9 H$; $\mathrm{df}=18, t=2.74, p=0.01$ ). Although these effects may not be indicative of changes in anxiety-like behavior or locomotor activity because control mice 

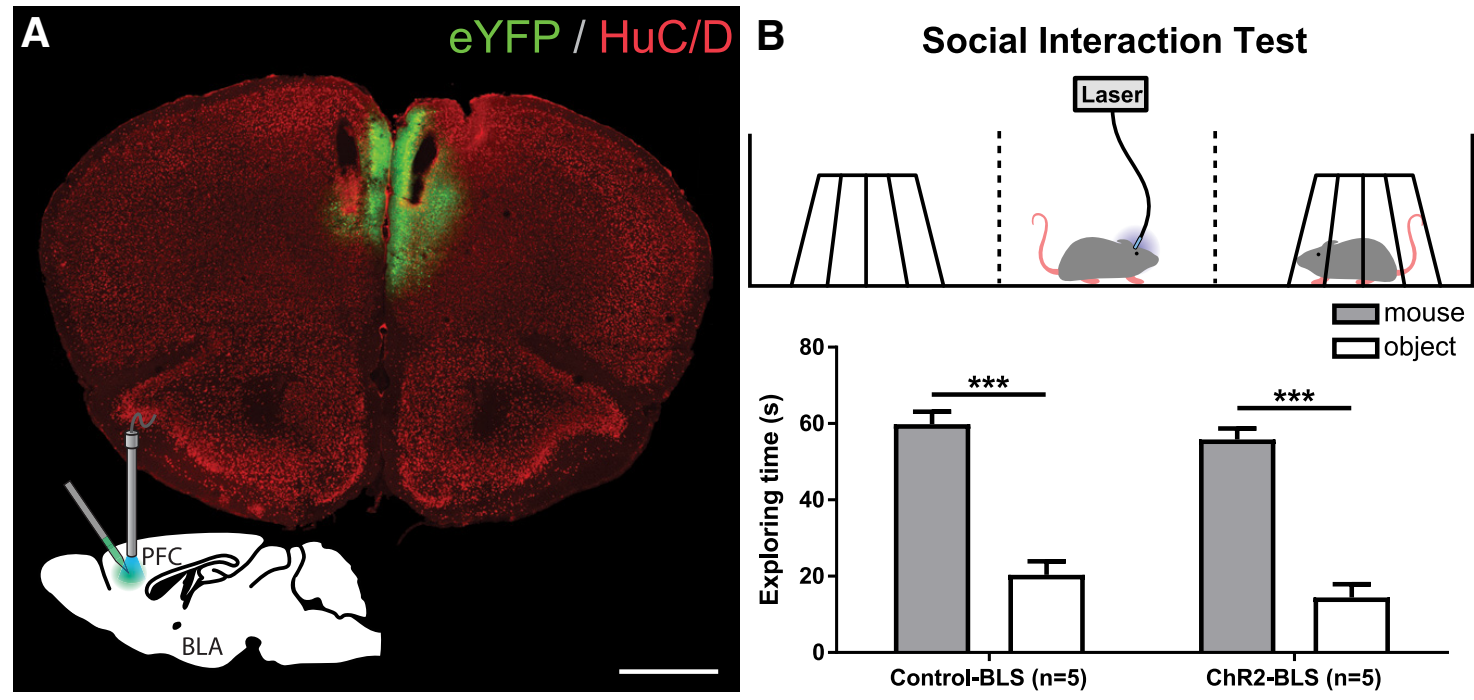

C

Social Recognition Test

D Novel Object Recognition Test
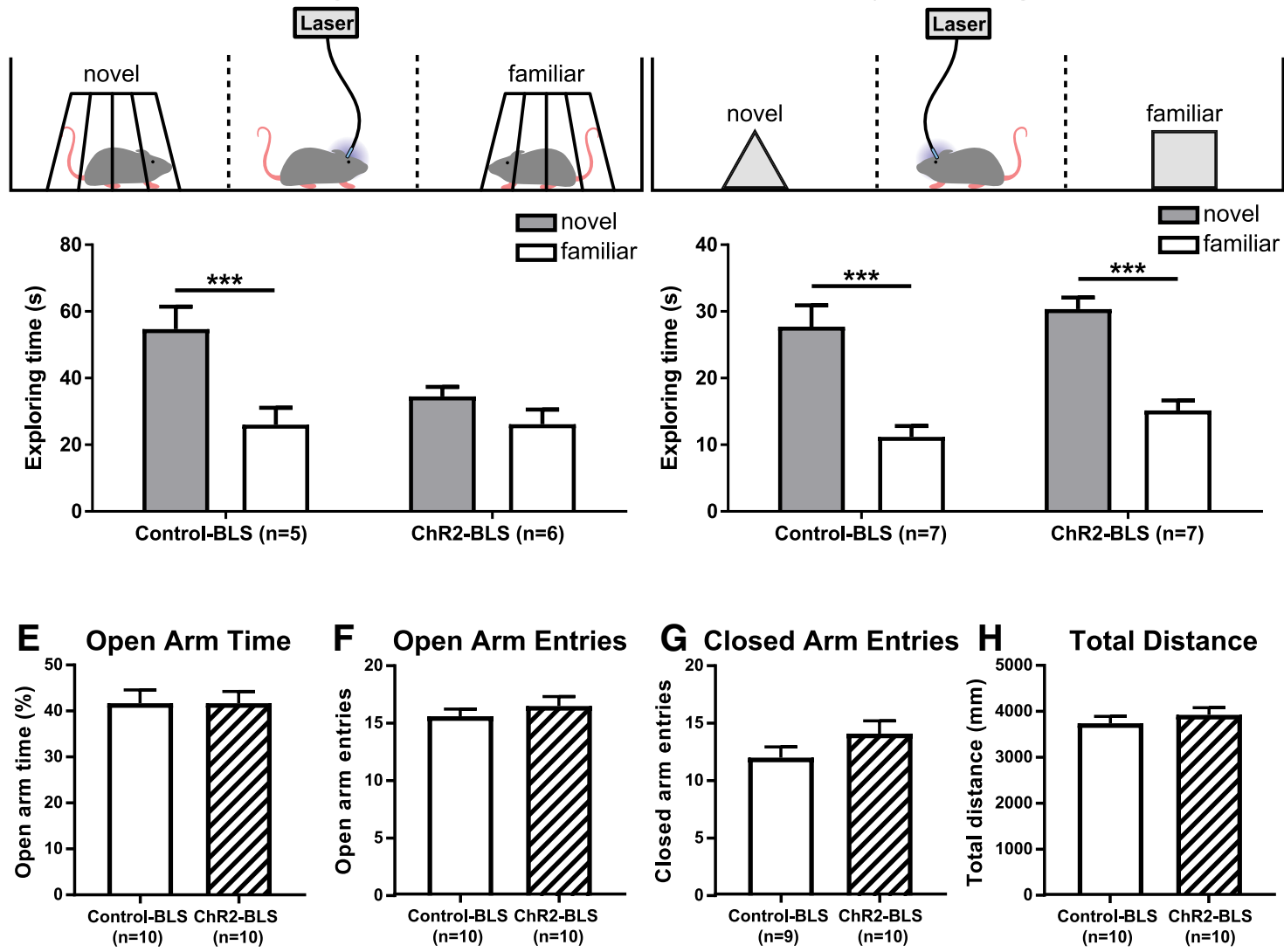

Figure 7. Effects of in vivo optogenetic activation of OXTR-expressing neurons in the PFC on sociability, social recognition, novel object recognition, and anxiety-like behavior. $A$, Coronal section through the PFC of an Oxtr-Cre mouse with AAV-eYFP injections and fiber optic implantations bilaterally targeting the PFC. Green represents eYFP. Red represents HuC/D. $\boldsymbol{B}$, Schematic and results of the three-chambered social test assessing time spent exploring a novel conspecific and an inanimate object. C, Social recognition test evaluating time spent exploring novel and familiar conspecifics. $\boldsymbol{D}$, Novel object recognition test evaluating preference to explore novel or familiar objects. $\boldsymbol{E}-\boldsymbol{H}$, Anxiety-like behavior and locomotion indicated by $(\boldsymbol{E})$ percentage of time spent in open arms, $(\boldsymbol{F})$ open arm entries, $(\boldsymbol{G})$ closed arm entries, and $(\boldsymbol{H})$ total distance traveled in the EPM. Scale bar: $\boldsymbol{A}, 500 \mu \mathrm{m} .{ }^{* * *} p<0.001$.

$(n=11)$ and those treated with AAV-ChR2-eYFP $(n=8)$ spent a similar percentage of time in the center of open field (control: $10.79 \pm 0.93 \%$, ChR2: $9.70 \pm 0.99 \% ; \mathrm{df}=17, t=0.79, p=0.44)$, and traveled similar distances (control: 16,003 $\pm 663.9 \mathrm{~mm}$, ChR2: $16,162 \pm 935.4 \mathrm{~mm}$; $\mathrm{df}=17, t=0.14, p=0.89$ ). These results, together, suggest that an oxytocin-sensitive connection from the PFC to the BLA is critically involved in the ability to distinguish novel from familiar conspecifics.

\section{Discussion}

Our experiments revealed a distinct population of neurons in the PFC that are depolarized by oxytocin and send direct excitatory glutamatergic projections to forebrain nuclei that are known to mediate social behavior. To the best of our knowledge, this is the first report of glutamatergic neurons in the PFC that express OXTR; and consequently, a variety of approaches were used for 

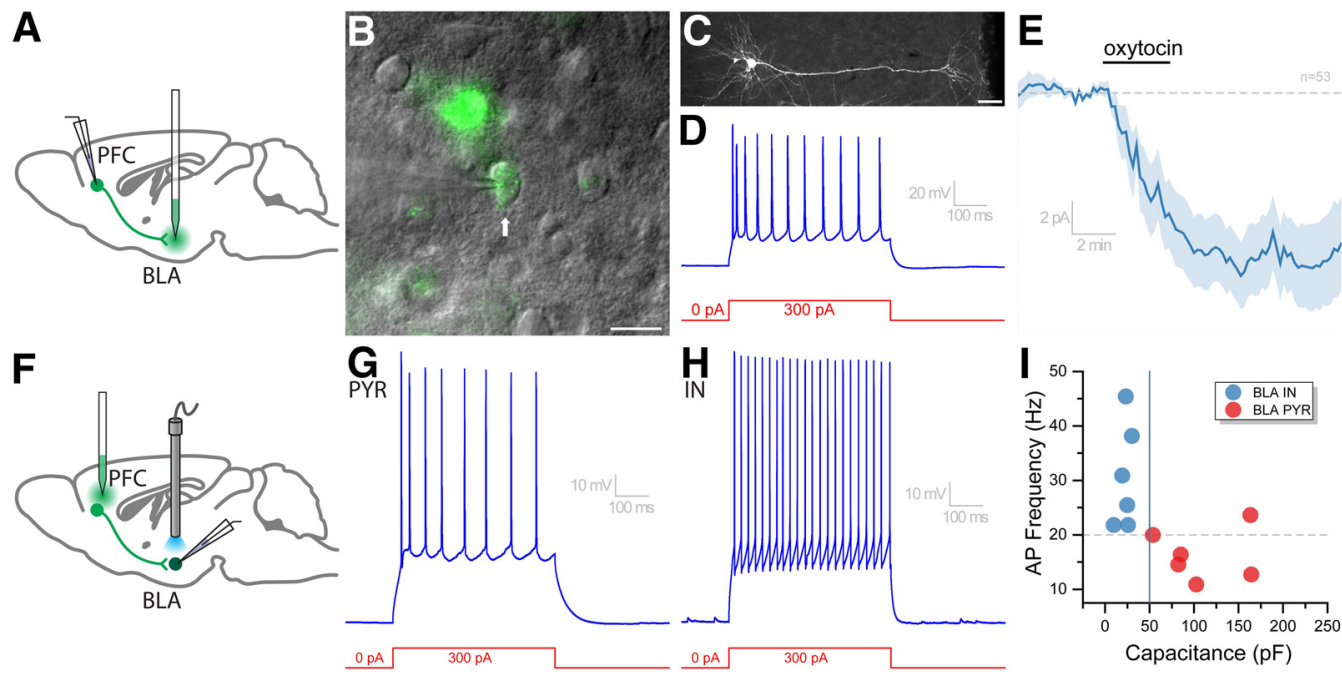

Figure 8. Oxytocin depolarizes BLA-projecting PFC neurons that release glutamate onto pyramidal (PYR) and interneurons (IN) in the BLA. $A$, Retro-fluorescent microspheres were injected into the BLA of WT mice to identify and obtain electrophysiological recordings from neurons in the PFC with direct projections to the BLA. $B$, Using epifluorescence with DIC microscopy, retrogradely labeled (green) PFC neurons were targeted for patch-clamp analysis. These neurons had $(\boldsymbol{C})$ morphology and $(\boldsymbol{D})$ firing properties like those of pyramidal neurons in Figure $2 K-L$. $\boldsymbol{E}$, Average response of all retrogradely labeled cells to oxytocin ( $3 \mathrm{~min}, 200-400 \mathrm{~nm} ; n=53$ ). $F$, Injection of Cre-inducible AAV-ChR2-eYFP into the PFC of Oxtr-Cre mice allows for CRACM in the BLA. G-I, BLS induced EPSCs in both small and large BLA neurons that exhibited a wide range of firing frequencies in response to a $300 \mathrm{pA}$ current injection. Among the light responsive BLA neurons, large capacitance slow firing cells were considered likely to by pyramidal neurons, whereas small capacitance faster firing cells were considered likely to be interneurons. Scale bars: $\boldsymbol{B}, 20 \mu \mathrm{m} ; \boldsymbol{C}, 50 \mu \mathrm{m}$.

their characterization. First, we conducted neuroanatomical experiments that demonstrated colocalization of eYFP with Oxtr mRNA and VGlut1 mRNA. These results, in conjunction with those from our opto-fMRI experiments, suggested that OXTRs are expressed on glutamatergic neurons that send long-range axonal projections to several subcortical nuclei. Complimentary electrophysiological experiments confirmed these results by demonstrating that OXTR-expressing neurons increased firing upon application of oxytocin, exhibited morphological and electrophysiological properties like those of pyramidal neurons, and in vitro optogenetic activation of their axons in mesolimbic targets elicited EPSCs that were abolished in the presence of glutamate receptor antagonists. Further, subsets of neurons in the PFC sending direct projections to the BLA were also found to be depolarized by oxytocin and in vitro optogenetic excitation of axons that arose from OXTR-expressing neurons in the PFC-elicited glutamate release onto pyramidal and interneurons residing in the BLA. Finally, in vivo optogenetic experiments discovered that selectively exciting this oxytocin-sensitive PFC to BLA connection abolished preference for social novelty without affecting sociability, preference for novel objects, or anxiety-like behavior. Together, our results suggest that OXTRs expressed on PFC neurons that project to the BLA may serve as phenotypic markers for a distinct neural circuit that is required for distinguishing between novel and familiar conspecifics.

While OXTRs may serve as phenotypic markers for neurons comprising this circuit, it is important to note that optogenetic excitation is an artificial manipulation and is not representative of how oxytocin may influence these neurons to affect behavior. Neuronal networks mediating social behavior include neurons that express OXTRs and inhibiting these receptors in the brain eliminates the ability to distinguish between novel and familiar conspecifics, suggesting that central OXTR signaling is necessary for social recognition (Ferguson et al., 2001; Lee et al., 2008a,b; Song et al., 2016). Differentiating novel from familiar conspecifics in the social recognition test likely requires synchronization of neuronal excitation and inhibition with social and spatial stimuli. Consistent with this notion, a recent study conducted in mice used in vivo calcium imaging with the three-chambered social interaction test to reveal that the preference for social interaction over object exploration is associated with temporal coupling of neuronal activity and social investigation (Murugan et al., 2017). One interpretation of these results is that, under normal conditions, endogenous oxytocin enables social recognition by coordinating the temporal and spatial coupling of social stimuli with neuronal activity. More than likely, optogenetic excitation of OXTR-expressing neurons in the PFC, as done in the present study, disrupts synchronization of neuronal activity with social and spatial stimuli, and consequently, impairs encoding of social memory.

Previous research suggests the possibility that OXTRs are expressed on astrocytes (Scala-Guenot and Strosser, 1992; Parent et al., 2008); however, the morphology and electrophysiological properties of virally transformed OXTR-expressing cells in the present study indicate a neuronal phenotype. Our studies focused mostly on glutamatergic neurons, but it is important to note that OXTR is also expressed by GABAergic neurons in the PFC. Prior studies went to great lengths to establish the phenotype of GABAergic interneurons in the PFC that express OXTR (Nakajima et al., 2014; Li et al., 2016). These studies found that, in the PFC of female mice, OXTRs are expressed on a unique population of somatostatin neurons that spontaneously fire upon application of oxytocin, and the activity of these neurons is required for social interest in male mice at the time of estrous (Nakajima et al., 2014). In the PFC of male mice, OXTR is also expressed on GABAergic interneurons that spontaneously fire upon application of oxytocin; however, these neurons produce corticotropin-releasing-hormone-binding protein, and their excitation reduces anxiety-like behavior, in part, by releasing GABA onto pyramidal neurons residing in layers $2 / 3$ and 5 of the PFC (Li et al., 2016). Consistent with this prior study, we found that OXTR is expressed on GABAergic interneurons in the PFC of male mice, and oxytocin similarly increased their spontaneous firing and in vitro optogenetic excitation elicited GABA release onto neighboring PFC neurons. However, our experiments using in vivo optogenetics did not observe the attenuation of anxiety-like behavior that was previously reported (Li et al., 2016), a discrepancy that may be due to our 

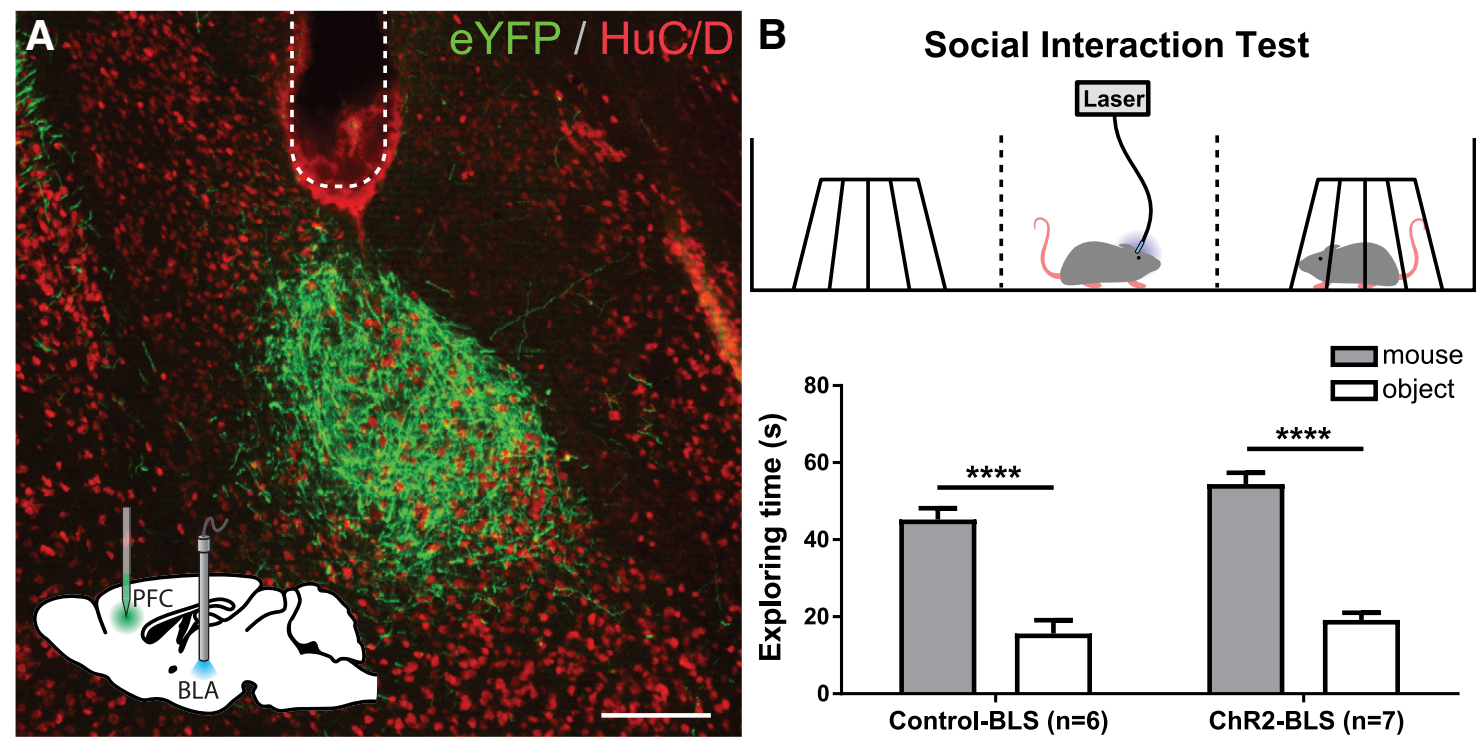

C Social Recognition Test

D Novel Object Recognition Test
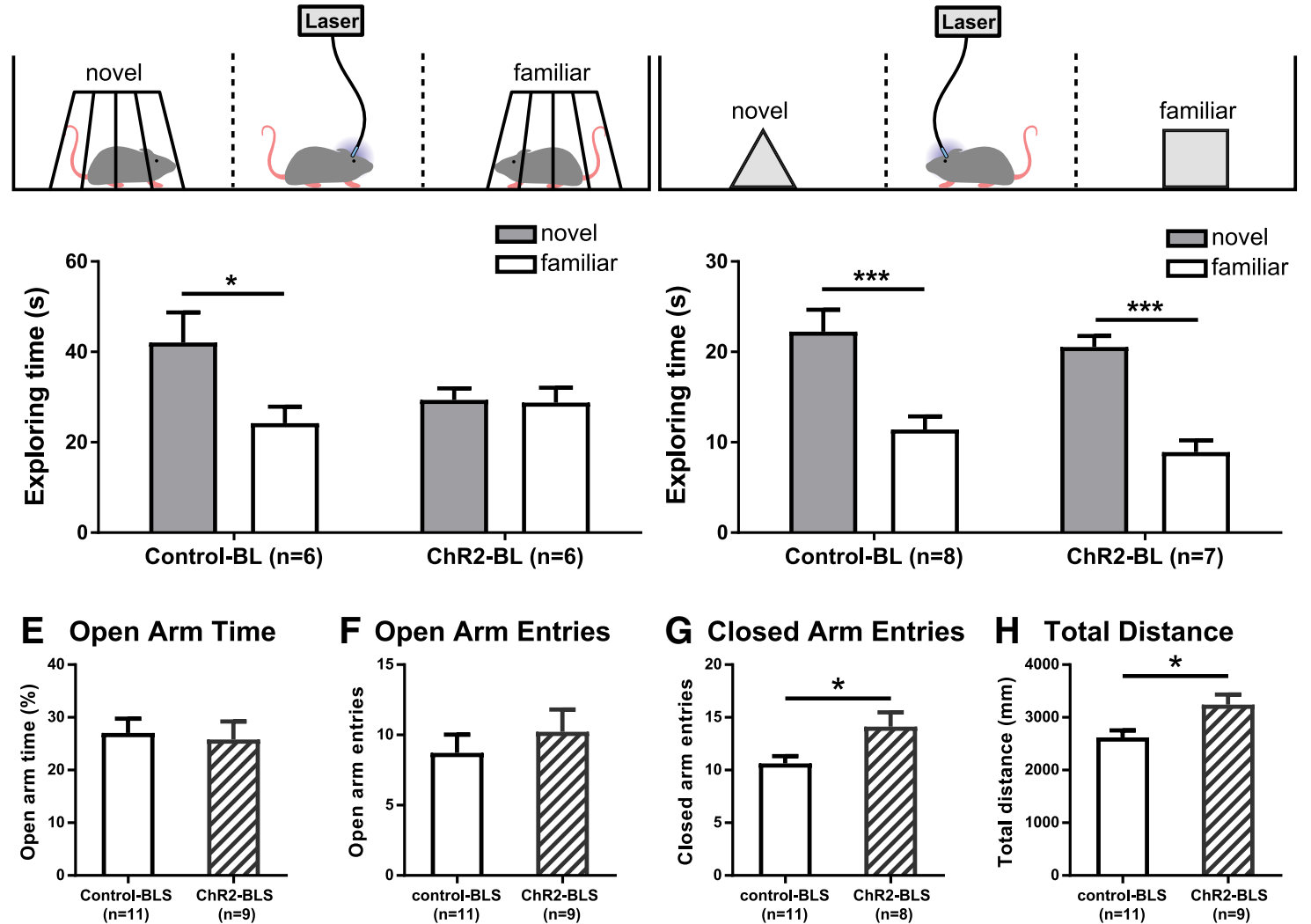

Figure 9. Optogenetic activation of axons in the BLA that originate from OXTR-expressing neurons in the PFC impairs social recognition. $A$, Inset, Schematic illustrating viral injections targeting OXTR-expressing neurons in the PFC with optical stimulation of their axons terminating in the BLA. $A$, Coronal section through the BLA of an Oxtr-Cre mouse given Cre-inducible AAV-ChR2-eYFP into the PFC and a chronic dwelling fiber optic targeting axons (eYFP; green) making appositions onto neurons (HuC/D; red) in the BLA. Dashed line indicates the placement of the fiber optic. $\boldsymbol{B}$, Schematic and results of the three-chambered social test assessing time spent exploring a novel conspecific and an inanimate object. C, Social recognition test evaluating time spent exploring novel and familiar conspecifics. $\boldsymbol{D}$, Novel object recognition test evaluating preference to explore a novel or familiar object. $\boldsymbol{E}-\boldsymbol{H}$, The effect of optogenetic excitation of axons in the BLA on anxiety-like behavior and locomotion, as indicated by $(\boldsymbol{E})$ percentage of time spent in open arms, $(\boldsymbol{F})$ open arm entries, $(\boldsymbol{G})$ closed arm entries, and $(\boldsymbol{H})$ total distance traveled in the EPM. Scale bar: $\boldsymbol{A}, 200 \mu \mathrm{m} .{ }^{*} p<0.05$. ${ }^{* * *} p<0.001$. ${ }^{* * *} p<0.0001$.

simultaneous excitation of both GABAergic and glutamatergic OXTR-expressing neurons in the PFC.

At first glance, the connectivity of OXTR-expressing glutamatergic neurons resembles those of CaMKII-producing neurons in the PFC that send glutamatergic projections to the NAc, BNST, amygdala, and VTA (Murugan et al., 2017). Optogenetic excitation of CaMKII-producing neurons in the PFC (Yizhar et al., 2011) or their projections to the NAc (Murugan et al., 2017) abolishes preference for sociability in the three-chambered social test. That is, mice with optogenetic activation of CaMKII- 
producing PFC neurons spend similar amounts of time investigating novel conspecifics and inanimate objects when given the opportunity to investigate both simultaneously (Yizhar et al., 2011; Murugan et al., 2017). In contrast, our study found that preference for sociability in the three-chambered social test was intact when OXTR-expressing neurons in the PFC or their projections to the BLA were optogenetically stimulated. One interpretation of these results is that neurons in the PFC that express OXTR also express CaMKII; however, seemingly not all CaMKIIproducing neurons in the PFC express OXTR because activation of the CaMKII-producing neurons causes impairments in social behavior that differ from what occurs when OXTR-expressing neurons are selectively activated. The implication is that, in the PFC, OXTRs are expressed on a subset of CaMKII-producing neurons that control preference for social novelty but not preference for sociability.

It is remarkable that optogenetic activation of OXTRexpressing neurons in the PFC impaired social recognition but had no effect on any of the other behaviors that were examined. These results are consistent with those from previous studies demonstrating that disrupting OXTR signaling with pharmacological, genetic, or optogenetic manipulations impairs learning within a social context but leaves nonsocial learning, such as novel object recognition or conditioned place preference for palatable food, completely intact (Choe et al., 2015; Raam et al., 2017). These studies found impairments in social learning when OXTRs were specifically inhibited in the olfactory cortex (Choe et al., 2015) or the hippocampus (Raam et al., 2017). These results, in conjunction with our own, suggest that a single brain nucleus does not mediate social learning. Instead, social learning very likely requires coordination of neuronal activity across networks that integrate sensory input, reward value, and vigilance to form socially relevant memories. In this regard, the PFC, hippocampus, and BLA have been heavily implicated in the control of social recognition (Garrido Zinn et al., 2016; Tanimizu et al., 2017), and it is likely that causing excitatory-inhibitory imbalance within OXTR-expressing neurons residing in any of these brain nuclei disrupts some aspect of social learning.

Neurodevelopment disorders, such as ASD, are associated with excitation-inhibition imbalance that alters the function of brain circuits controlling social behavior, which may create deficits in the processing of socially relevant stimuli (Tyzio et al., 2006, 2014; Nelson and Valakh, 2015). A hallmark of ASD is difficulty performing tasks that require face recognition, which is the ability to identify a person based on remembering facial features (Weigelt et al., 2012; Ewbank et al., 2017). The difficulties with facial recognition appear to be specific because persons with ASD perform similarly to typical subjects when remembering geometric shapes or different types of automobiles (Ewbank et al., 2017). In typical subjects, repeatedly viewing the same face is associated with decreased neural activity within subdivisions of the PFC (Grill-Spector and Malach, 2001; Andrews and Ewbank, 2004), and interestingly, this decrease is attenuated in subjects with ASD (Ewbank et al., 2017). This symptomology is particularly intriguing because it is reminiscent of the impaired social recognition but intact novel object recognition that occurred with optogenetic excitation of OXTR-expressing neurons in the PFC. Consequently, it is tempting to speculate that the neurocircuitry revealed in our study may become compromised with neurological disorders, such as ASD; however, additional experimentation is required to support or refute this hypothesis.

\section{References}

Amadei EA, Johnson ZV, Jun Kwon Y, Shpiner AC, Saravanan V, Mays WD, Ryan SJ, Walum H, Rainnie DG, Young LJ, Liu RC (2017) Dynamic corticostriatal activity biases social bonding in monogamous female prairie voles. Nature 546:297-301.

Andrews TJ, Ewbank MP (2004) Distinct representations for facial identity and changeable aspects of faces in the human temporal lobe. Neuroimage 23:905-913.

Berridge KC, Kringelbach ML (2015) Pleasure systems in the brain. Neuron 86:646-664.

Bluthé RM, Gheusi G, Dantzer R (1993) Gonadal steroids influence the involvement of arginine vasopressin in social recognition in mice. Psychoneuroendocrinology 18:323-335.

Brennan PA, Kendrick KM (2006) Mammalian social odours: attraction and individual recognition. Philos Trans $\mathrm{R}$ Soc Lond B Biol Sci 361:2061-2078.

Bukhari Q, Schroeter A, Rudin M (2018) Increasing isoflurane dose reduces homotopic correlation and functional segregation of brain networks in mice as revealed by resting-state fMRI. Sci Rep 8:10591.

Chen P, Hong W (2018) Neural circuit mechanisms of social behavior. Neuron 98:16-30.

Chini B, Verhage M, Grinevich V (2017) The action radius of oxytocin release in the mammalian CNS: from single vesicles to behavior. Trends Pharmacol Sci 38:982-991.

Choe HK, Reed MD, Benavidez N, Montgomery D, Soares N, Yim YS, Choi GB (2015) Oxytocin mediates entrainment of sensory stimuli to social cues of opposing valence. Neuron 87:152-163.

Colon-Perez LM, Tran K, Thompson K, Pace MC, Blum K, Goldberger BA, Gold MS, Bruijnzeel AW, Setlow B, Febo M (2016) The psychoactive designer drug and bath salt constituent MDPV causes widespread disruption of brain functional connectivity. Neuropsychopharmacology 41:2352-2365.

Cox RW (1996) AFNI: software for analysis and visualization of functional magnetic resonance neuroimages. Comput Biomed Res 29:162-173.

Dana H, Chen TW, Hu A, Shields BC, Guo C, Looger LL, Kim DS, Svoboda K (2014) Thyl-GCaMP6 transgenic mice for neuronal population imaging in vivo. PLoS One 9:e108697.

Dantzer R (1998) Vasopressin, gonadal steroids and social recognition. Prog Brain Res 119:409-414

Davis M, Walker DL, Miles L, Grillon C (2010) Phasic vs sustained fear in rats and humans: role of the extended amygdala in fear vs anxiety. Neuropsychopharmacology 35:105-135.

de Kloet AD, Pitra S, Wang L, Hiller H, Pioquinto DJ, Smith JA, Sumners C, Stern JE, Krause EG (2016) Angiotensin type-2 receptors influence the activity of vasopressin neurons in the paraventricular nucleus of the hypothalamus in male mice. Endocrinology 157:3167-3180.

Elands J, Beetsma A, Barberis C, de Kloet ER (1988) Topography of the oxytocin receptor system in rat brain: an autoradiographical study with a selective radioiodinated oxytocin antagonist. J Chem Neuroanat 1:293-302.

Ewbank MP, Pell PJ, Powell TE, von dem Hagen EA, Baron-Cohen S, Calder AJ (2017) Repetition suppression and memory for faces is reduced in adults with autism spectrum conditions. Cereb Cortex 27:92-103.

Ferguson JN, Aldag JM, Insel TR, Young LJ (2001) Oxytocin in the medial amygdala is essential for social recognition in the mouse. J Neurosci 21: $8278-8285$

Franklin KB, Paxinos G (2008) The mouse brain in stereotaxic coordinates, Ed 3. Amsterdam: Elsevier/Academic.

Garrido Zinn C, Clairis N, Silva Cavalcante LE, Furini CR, de Carvalho Myskiw J, Izquierdo I (2016) Major neurotransmitter systems in dorsal hippocampus and basolateral amygdala control social recognition memory. Proc Natl Acad Sci U S A 113:E4914-E4919.

Grill-Spector K, Malach R (2001) fMR-adaptation: a tool for studying the functional properties of human cortical neurons. Acta Psychol (Amst) 107:293-321.

Jenkinson M, Bannister P, Brady M, Smith S (2002) Improved optimization for the robust and accurate linear registration and motion correction of brain images. Neuroimage 17:825-841.

Knobloch HS, Charlet A, Hoffmann LC, Eliava M, Khrulev S, Cetin AH, Osten P, Schwarz MK, Seeburg PH, Stoop R, Grinevich V (2012) Evoked axonal oxytocin release in the central amygdala attenuates fear response. Neuron 73:553-566.

Landgraf R, Neumann ID (2004) Vasopressin and oxytocin release within 
the brain: a dynamic concept of multiple and variable modes of neuropeptide communication. Front Neuroendocrinol 25:150-176.

Lee HJ, Caldwell HK, Macbeth AH, Young WS 3rd (2008a) Behavioural studies using temporal and spatial inactivation of the oxytocin receptor. Prog Brain Res 170:73-77.

Lee HJ, Caldwell HK, Macbeth AH, Tolu SG, Young WS 3rd (2008b) A conditional knockout mouse line of the oxytocin receptor. Endocrinology 149:3256-3263.

Lefevre A, Sirigu A (2016) The two fold role of oxytocin in social developmental disorders: a cause and a remedy? Neurosci Biobehav Rev 63:168-176.

Li K, Nakajima M, Ibañez-Tallon I, Heintz N (2016) A cortical circuit for sexually dimorphic oxytocin-dependent anxiety behaviors. Cell 167:60-72.e11.

LoParo D, Waldman ID (2015) The oxytocin receptor gene (OXTR) is associated with autism spectrum disorder: a meta-analysis. Mol Psychiatry 20:640-646.

Murugan M, Jang HJ, Park M, Miller EM, Cox J, Taliaferro JP, Parker NF, Bhave V, Hur H, Liang Y, Nectow AR, Pillow JW, Witten IB (2017) Combined social and spatial coding in a descending projection from the prefrontal cortex. Cell 171:1663-1677.e16.

Nakajima M, Görlich A, Heintz N (2014) Oxytocin modulates female sociosexual behavior through a specific class of prefrontal cortical interneurons. Cell 159:295-305.

Nelson SB, Valakh V (2015) Excitatory/inhibitory balance and circuit homeostasis in autism spectrum disorders. Neuron 87:684-698.

Oettl LL, Ravi N, Schneider M, Scheller MF, Schneider P, Mitre M, da Silva Gouveia M, Froemke RC, Chao MV, Young WS, Meyer-Lindenberg A, Grinevich V, Shusterman R, Kelsch W (2016) Oxytocin enhances social recognition by modulating cortical control of early olfactory processing. Neuron 90:609-621.

Parent A-S, Rasier G, Matagne V, Lomniczi A, Lebrethon M-C, Gérard A, Ojeda SR, Bourguignon J-P (2008) Oxytocin facilitates female sexual maturation through a glia-to-neuron signaling pathway. Endocrinology 149:1358-1365.

Raam T, McAvoy KM, Besnard A, Veenema AH, Sahay A (2017) Hippocampal oxytocin receptors are necessary for discrimination of social stimuli. Nat Commun 8:2001.

Sabihi S, Durosko NE, Dong SM, Leuner B (2014) Oxytocin in the prelimbic medial prefrontal cortex reduces anxiety-like behavior in female and male rats. Psychoneuroendocrinology 45:31-42.

Scala-Guenot DD, Strosser M (1992) Oxytocin receptors on cultured astroglial cells. Kinetic and pharmacological characterization of oxytocinbinding sites on intact hypothalamic and hippocampic cells from foetal rat brain. Biochem J 284:491-497.

Schindelin J, Arganda-Carreras I, Frise E, Kaynig V, Longair M, Pietzsch T, Preibisch S, Rueden C, Saalfeld S, Schmid B, Tinevez JY, White DJ,
Hartenstein V, Eliceiri K, Tomancak P, Cardona A (2012) Fiji: an opensource platform for biological-image analysis. Nat Methods 9:676-682.

Schneider CA, Rasband WS, Eliceiri KW (2012) NIH image to ImageJ: 25 years of image analysis. Nat Methods 9:671-675.

Silverman JL, Yang M, Lord C, Crawley JN (2010) Behavioural phenotyping assays for mouse models of autism. Nat Rev Neurosci 11:490-502.

Smeltzer MD, Curtis JT, Aragona BJ, Wang Z (2006) Dopamine, oxytocin, and vasopressin receptor binding in the medial prefrontal cortex of monogamous and promiscuous voles. Neurosci Lett 394:146-151.

Sofroniew MV (1983) Morphology of vasopressin and oxytocin neurones and their central and vascular projections. Prog Brain Res 60:101-114.

Song Z, Larkin TE, Malley MO, Albers HE (2016) Oxytocin (OT) and arginine-vasopressin (AVP) act on OT receptors and not AVP Vla receptors to enhance social recognition in adult Syrian hamsters (Mesocricetus auratus). Horm Behav 81:20-27.

Stoop R (2012) Neuromodulation by oxytocin and vasopressin. Neuron $76: 142-159$

Swanson LW, Sawchenko PE (1983) Hypothalamic integration: organization of the paraventricular and supraoptic nuclei. Annu Rev Neurosci 6:269-324.

Tanimizu T, Kenney JW, Okano E, Kadoma K, Frankland PW, Kida S (2017) Functional connectivity of multiple brain regions required for the consolidation of social recognition memory. J Neurosci 37:4103-4116.

Tyzio R, Cossart R, Khalilov I, Minlebaev M, Hübner CA, Represa A, Ben-Ari Y, Khazipov R (2006) Maternal oxytocin triggers a transient inhibitory switch in GABA signaling in the fetal brain during delivery. Science 314:1788-1792.

Tyzio R, Nardou R, Ferrari DC, Tsintsadze T, Shahrokhi A, Eftekhari S, Khalilov I, Tsintsadze V, Brouchoud C, Chazal G, Lemonnier E, Lozovaya N, Burnashev N, Ben-Ari Y (2014) Oxytocin-mediated GABA inhibition during delivery attenuates autism pathogenesis in rodent offspring. Science 343:675-679.

Weigelt S, Koldewyn K, Kanwisher N (2012) Face identity recognition in autism spectrum disorders: a review of behavioral studies. Neurosci Biobehav Rev 36:1060-1084.

Yizhar O, Fenno LE, Prigge M, Schneider F, Davidson TJ, O'Shea DJ, Sohal VS, Goshen I, Finkelstein J, Paz JT, Stehfest K, Fudim R, Ramakrishnan C, Huguenard JR, Hegemann P, Deisseroth K (2011) Neocortical excitation/inhibition balance in information processing and social dysfunction. Nature 477:171-178.

Zhou Y, Kaiser T, Monteiro P, Zhang X, Van der Goes MS, Wang D, Barak B, Zeng M, Li C, Lu C, Wells M, Amaya A, Nguyen S, Lewis M, Sanjana N, Zhang M, Zhang F, Fu Z, Feng G (2016) Mice with Shank3 mutations associated with ASD and schizophrenia display both shared and distinct defects. Neuron 89:147-162. 